\title{
EXPECTED MAKESPAN MINIMIZATION ON IDENTICAL MACHINES IN TWO INTERCONNECTED QUEUES
}

\author{
TARA JAVIDI \\ EECS Department \\ The University of Michigan \\ Ann Arbor, MI 48109-2122 \\ E-mail: taraj@eecs.umich.edu \\ NAH-OAK SONG \\ Advanced Network Technologies Division \\ National Institute of Standards and Technology \\ Gaithersburg, MD 20899-8920 \\ E-mail: nsong@antd.nist.gov \\ Demosthenis TeneKetzis \\ EECS Department \\ The University of Michigan \\ Ann Arbor, MI 48109-2122 \\ E-mail: teneketzis@eecs.umich.edu
}

\begin{abstract}
We study the scheduling of jobs in a system of two interconnected service stations, called $Q_{1}$ and $Q_{2}$, on $m(m \geq 2)$ identical machines available in parallel, so that every machine can process any jobs in $Q_{1}$ or $Q_{2}$. Preemption is allowed. Under certain conditions on the arrival, service, and interconnection processes, we determine a scheduling policy that minimizes the expected makespan.
\end{abstract}

\section{INTRODUCTION: PROBLEM FORMULATION}

We investigate the following makespan minimization problem in two interconnected queues with arrivals to the first queue. 
Problem $(P)$ : Consider a system of two interconnected queues as in Figure 1. Initially, there are $N_{i}$ jobs in the queue $i\left(Q_{i}\right), i=1,2$. New jobs arrive in the first queue according to a Poisson process with the parameter $\lambda$. There are $m$ identical machines available in parallel, so that every machine can process any job in $Q_{1}$ and $Q_{2}$. The job processing times in $Q_{1}$ and $Q_{2}$ are exponentially distributed random variables with rates $\mu_{1}$ and $\mu_{2}$, respectively. After a job completes service in $Q_{1}$, it either joins $Q_{2}$ with probability $p$ or leaves the system with probability $1-p$. When a job initially in $Q_{1}$ joins $Q_{2}$, it creates $k$ jobs in $Q_{2}$. Whenever a job completes service in $Q_{2}$, it leaves the system. Preemption is allowed. The objective is to determine a server allocation policy that minimizes the expected value of the first time the system is empty (i.e., expected makespan).

The above problem is motivated by detection and classification issues in automated target recognition (ATR) systems and by quality control issues in wafer fabrication.

The ability to detect and classify objects in a timely fashion is a critical factor in determining the efficiency of ATR systems. One approach to targeting detection and classification in ATR systems is the following: The area under surveillance is divided into subpatches. Initially, each subpatch is processed separately using certain sequential detection and classification rules. One of the following situations can arise after the subpatch processing is completed: (1) It is determined that there is no target in the subpatch; in this situation the subpatch is discarded and is not processed any further. (2) It is determined that there are targets of potential interest in the subpatch; in this situation, the subpatch is divided into $k(k \geq 2)$ smaller subpatches and a separate processor is allocated to each of the newly created subpatches. This process is repeated until all potential targets are detected and classified. Under the assumption that there is a fixed number of resources (e.g., antennas and their associated data processing systems) and a fixed set of subpatch processing algorithms (e.g., algorithms used for detection and classification of each stage of the above process), the objective is to determine resource allocation strategies to minimize the expected length of surveillance time. This is equivalent to minimizing the expected makespan (i.e., the expected value of the first time that the system is empty). Problem (P) captures essential features of this problem as follows. The number of tasks initially present in $Q_{1}$ corresponds to the number of subpatches originally available

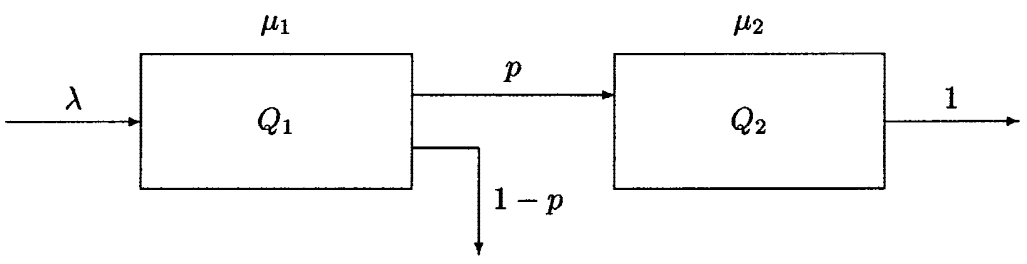

Figure 1. Two interconnected queues in Problem (P). 
for processing. Initially, we have $N_{2}=0$. Each task that completes processing in $Q_{1}$ and leaves the system corresponds to a subpatch, the processing of which is completed and no target is found. Each task that completes processing in $Q_{1}$ and then joins $Q_{2}$ and creates $k$ new tasks in $Q_{2}$ corresponds to a subpatch that is further subdivided into $k$ new smaller subpatches. There are no external new arrivals; hence, $\lambda=0$.

Problem (P) arises also in semiconductor manufacturing systems, specifically in quality control of wafer fabrication. A two-stage inspection is used to speed up the quality control process in wafer fabrication. The first stage sorts out, through a preliminary test, wafers that are defective and discards them. The remaining wafers go through a second inspection; by the end of that inspection, they are either declared perfect (and they can be used in logic chips, memory chips, etc.) or discarded. The above two-stage inspection can be described by the system of Figure 1 as follows. New wafers that arrive in the system for inspection join $Q_{1}$. The preliminary stage of inspection can be modeled by the processing of a wafer in $Q_{1}$. The wafers that are found imperfect after the first inspection (i.e., after their processing in $Q_{1}$ ) are discarded (i.e., they leave the system). The wafers that pass the first inspection join $Q_{2}$ and go through the second inspection. The quality control process is concluded after the processing in $Q_{2}$ (i.e., the wafers leave the system of Figure 1 after their second test is completed). A reasonable objective for this problem is to minimize the length of busy cycles. In our model, this can be translated into minimizing the first time that the system is empty. By the memoryless property of the arrival process, every time the system gets empty, the system generates a statistically similar process. As a result, minimizing the expected makespan, as the first time that the system is empty, is a reasonable goal.

So far, makespan minimization problems have been investigated either for systems of serial machines or on parallel machines (see $[1,8,9,13-15,18]$, and the references therein). Problem $(\mathrm{P})$ is distinctly different from the makespan minimization problems on serial machines, known as shop scheduling (see [8,9]). Traditionally, makespan minimization problems on identical parallel machines are formulated as follows. There are $N$ jobs to be processed by $m$ identical machines $(m<N)$. These machines are available in parallel, so that every machine can process any job. The jobs' processing times are, in general, random, and the objective is to find a strategy that minimizes the expected makespan. Several variations of the above problem with different assumption on the jobs' processing times have been investigated (see, e.g., $[1,8,13-15])$. The main result states that in the above problem, it is optimal to serve the jobs with the longest expected processing time (LEPT) first. The problems in makespan minimization on parallel machines investigated so far are fundamentally different from Problem (P) for the following reason. In the traditional formulation, the number of jobs in the system is monotonically decreasing in the time intervals between successive arrivals. An increase in the number of jobs in the system occurs only at the arrival of the new jobs; these times are independent of the service completion times. In Problem (P), with $k>1$, we have an increase in the number of the jobs to be processed at the completion times if the interconnection 
occurs. Thus, the time instants where the number of jobs in the system increases are highly correlated with job completion times in $Q_{1}$. The correlation between job arrivals to $Q_{2}$ and job completions in $Q_{1}$ gives rise to problems that are conceptually different from those previously studied, even when $k=1$. In the case $k=1$, it might appear that one can convert Problem (P) into a traditional makespan minimization problem on parallel machines where the jobs' processing times are described by a random variable $T_{c}$, which with a probability $1-p$, is exponential with parameter $\mu_{1}$ and, with probability $p$, is equal to the sum of two independent exponential random variables with parameters $\mu_{1}$ and $\mu_{2}$. Such a transformation does not result in a problem equivalent to Problem (P) because it discards some of the information available in Problem (P). Specifically, such a transformation ignores the information about the occurrence of a job completion in $Q_{1}$ and its interconnection to $Q_{2}$. More precisely, if $T_{i}$ denotes the service time in $Q_{i}, i=1,2$, and $I$ denotes the indicator function for the interconnection, then

$$
\sigma\left(T_{c}\right) \subset \sigma\left(T_{1}\right) \vee \sigma\left(T_{2}\right) \vee \sigma(I)
$$

with strict inclusion and where $\sigma\left(T_{1}\right) \vee \sigma\left(T_{2}\right) \vee \sigma(I)$ is the minimal $\sigma$-field with respect to which $T_{1}, T_{2}$, and $I$ are all measurable. Note that this loss of information implies that an optimal solution to the transformed problem is not guaranteed to be an optimal solution to Problem (P).

From the above discussion, it is evident that Problem $(\mathrm{P})$ is distinctly different from traditional makespan minimization problems in $[1,8,9,13-15,18]$. It is also different from [11] because it includes new arrivals.

Problem (P) can be viewed as scheduling of a random task graph where the tasks' processing times are identically distributed at each "generation" (cf. [3,5]), but are statistically different across different generations. In this aspect, Problem (P) has features similar to those of [3-7], but is distinctly different from the problems investigated in [3-7] for the following reasons. In [3], the tasks' processing times are deterministic. In $[4,6,7]$, the graphs are deterministic. In [5], which is an unpublished document, the processing times at all generations of the graph are identically distributed. In our opinion, the exchange arguments leading to the results of $[3,5]$ depend heavily on the assumption that the processing times are either deterministic or identically distributed across "generations" and cannot be extended in a straightforward manner to the situation in which the processing times are not statistically identical across generations. For the special case when the processing times are statistically identical (i.e., $\mu_{1}=\mu_{2}$ ), the result of this article is the same as that of [5].

The main contribution of this article is the analysis of Problem (P). As demonstrated by the example of Section 3, the nature of an optimal allocation policy changes as the parameters $\mu_{1}, \mu_{2}$, and $p$ vary. We do not attempt to determine an optimal allocation policy for all possible combinations of values of $\mu_{1}, \mu_{2}$, and $p$. Instead, we consider a specific policy $g^{*}$ that gives priority to $Q_{1}$ and we establish a condition on $\mu_{1}, \mu_{2}$, and $p$ sufficient to guarantee that $g^{*}$ is optimal. Interestingly, this condition guarantees that policy $g^{*}$ coincides with LEPT. Furthermore, when $k=1$, we prove 
that if the above condition is not satisfied, then it is always optimal to give priority to $Q_{2}$. This, again, coincides with LEPT for case when $k=1$.

The article is organized as follows. The main result on the optimality of policy $g^{*}$ and its proof are stated in Section 2. In Section 2.2, we introduce the functional equation of this problem and transform and reduce this equation into a form that can be analyzed, using the iteration method. In Section 2.3, an induction proof of the optimality of $g^{*}$ under the specified sufficient condition is given. In Section 3, we establish a very simple example to study the necessity and sufficiency of condition 2 ; in this Section, we discuss the special case $k=1$ and prove that, for $k=1$, if the condition in Section 2 is not satisfied, the policy that gives priority to $Q_{2}$ is optimal.

\section{ANALYSIS OF PROBLEM (P)}

\subsection{The Main Result}

In this section, we provide the main result of the article. We establish a condition on the processing times in $Q_{1}$ and $Q_{2}$ and the interconnection process $(p)$ sufficient to guarantee the optimality of policy $g^{*}$ (i.e., the policy that gives priority to $Q_{1}$ ). In contrast to the condition presented in [11], our condition is independent of the number $m$ of machines and the number $k$ of jobs created in $Q_{2}$ after an interconnection. Note that the nature of the optimality is interesting only when the expected makespan is finite. In order to assure the finiteness of makespan, we assume that $1 / \mu_{1}+$ $k / \mu_{2}<m / \lambda$ or, equivalently, $m \mu_{1} \mu_{2} / \lambda\left(\mu_{2}+k \mu_{1}\right)>1$. Under this condition, we can show that even a suboptimal policy like FIFO has a finite expected makespan. The argument is similar to Whitt's proof in [16].

The following theorem gives the main results of the article.

Theorem 1: Assume $m \mu_{1} \mu_{2} / \lambda\left(\mu_{2}+k \mu_{1}\right)>1$. Policy $g^{*}$ (i.e., the policy that gives priority to $Q_{1}$ ) is optimal for Problem $(P)$ under the following condition:

$$
p \geq \frac{\mu_{1}-\mu_{2}}{\mu_{1}} .
$$

We proceed to establish the main result of the article as follows: First, via some preliminary analysis, we show that Problem $(\mathrm{P})$ can be reduced to a corresponding $n$-stage problem. By induction, we establish the optimality of policy $g^{*}$ for the corresponding $n$-stage problem under the conditions of Theorem 1 . The philosophy of our approach is quite similar to that of [18].

We note that the condition of Theorem 1 is automatically satisfied if $\mu_{1} \leq \mu_{2}$ or $p=1$.

\subsection{Reduction of Problem (P) to an $n$-Stage Problem}

We can formulate Problem (P) as a Markov decision problem. The state space in our model consists of the ordered pairs of the form $\left(x_{1}, x_{2}\right)$, where $x_{i}$ is the number of available jobs in $Q_{i}$. The action space $A_{\left(x_{1}, x_{2}\right)}$ at state $\left(x_{1}, x_{2}\right)$ consists of the ordered 
pairs of the form $\left(g_{1}\left(x_{1}, x_{2}\right), g_{2}\left(x_{1}, x_{2}\right)\right)$, where $g_{i}\left(x_{1}, x_{2}\right)$ are the possible allocation of servers to the jobs in $Q_{i}$. Without loss of generality, we can restrict our attention to the Markov policies denoted by the vector $g=\left(g_{1}\left(x_{1}, x_{2}\right), g_{2}\left(x_{1}, x_{2}\right)\right)$ for which

$$
g_{1}\left(x_{1}, x_{2}\right)+g_{2}\left(x_{1}, x_{2}\right)=\min \left(x_{1}+x_{2}, m\right) .
$$

Given the state $\left(x_{1}, x_{2}\right)$ and the action $\left(g_{1}\left(x_{1}, x_{2}\right), g_{2}\left(x_{1}, x_{2}\right)\right)$, the time until the next transition is an exponential random variable with parameter $g_{1}\left(x_{1}, x_{2}\right) \mu_{1}+$ $g_{2}\left(x_{1}, x_{2}\right) \mu_{2}+\lambda$. As a result, the next state can be one of the following states with the corresponding probabilities:

$$
\begin{aligned}
& \left(x_{1}+1, x_{2}\right) \quad \text { with probability } \lambda /\left(g_{1}\left(x_{1}, x_{2}\right) \mu_{1}+g_{2}\left(x_{1}, x_{2}\right) \mu_{2}+\lambda\right) \\
& \left(x_{1}-1, x_{2}+1\right) \text { with probability } \frac{p \mu_{1} g_{1}\left(x_{1}, x_{2}\right)}{g_{1}\left(x_{1}, x_{2}\right) \mu_{1}+g_{2}\left(x_{1}, x_{2}\right) \mu_{2}+\lambda} \\
& \left(x_{1}-1, x_{2}\right) \quad \text { with probability } \frac{(1-p) \mu_{1} g_{1}\left(x_{1}, x_{2}\right)}{g_{1}\left(x_{1}, x_{2}\right) \mu_{1}+g_{2}\left(x_{1}, x_{2}\right) \mu_{2}+\lambda} \\
& \left(x_{1}, x_{2}-1\right) \quad \text { with probability } \frac{\mu_{2} g_{2}\left(x_{1}, x_{2}\right)}{g_{1}\left(x_{1}, x_{2}\right) \mu_{1}+g_{2}\left(x_{1}, x_{2}\right) \mu_{2}+\lambda} .
\end{aligned}
$$

Denote by $V\left(x_{1}, x_{2}\right)$ the minimum expected makespan. Then, $V$ satisfies the dynamic programming equation

$$
\begin{aligned}
V\left(x_{1}, x_{2}\right)=\min _{g}\{ & \left(1+\mu_{1} g_{1}\left(x_{1}, x_{2}\right)\left[p V\left(x_{1}-1, x_{2}+k\right)+(1-p) V\left(x_{1}-1, x_{2}\right)\right]\right. \\
& +\mu_{2} g_{2}\left(x_{1}, x_{2}\right) V\left(x_{1}, x_{2}-1\right) \\
& \left.\left.+\lambda V\left(x_{1}+1, x_{2}\right)\right) /\left(\mu_{1} g_{1}\left(x_{1}, x_{2}\right)+\mu_{2} g_{2}\left(x_{1}, x_{2}\right)+\lambda\right)\right\}
\end{aligned}
$$

with $V(0,0)=0$. Equation (2) ensures that for every $g=\left(g_{1}\left(x_{1}, x_{2}\right), g_{2}\left(x_{1}, x_{2}\right)\right)$,

$$
\begin{aligned}
0 \leq & 1+\mu_{1} g_{1}\left(x_{1}, x_{2}\right)\left[p V\left(x_{1}-1, x_{2}+k\right)+(1-p) V\left(x_{1}-1, x_{2}\right)\right] \\
& +\mu_{2} g_{2}\left(x_{1}, x_{2}\right) V\left(x_{1}, x_{2}-1\right)-\left(\mu_{1} g_{1}\left(x_{1}, x_{2}\right)+\mu_{2} g_{2}\left(x_{1}, x_{2}\right)+\lambda\right) V\left(x_{1}, x_{2}\right) \\
& +\lambda V\left(x_{1}+1, x_{2}\right)
\end{aligned}
$$

and is equivalent to

$$
\begin{aligned}
V\left(x_{1}, x_{2}\right)=\min _{g}\{ & \frac{1}{R}+\frac{\mu_{1} g_{1}\left(x_{1}, x_{2}\right)}{R}\left[p V\left(x_{1}-1, x_{2}+k\right)+(1-p) V\left(x_{1}-1, x_{2}\right)\right] \\
& +\frac{\mu_{2} g_{2}\left(x_{1}, x_{2}\right)}{R} V\left(x_{1}, x_{2}-1\right)+\frac{\lambda}{R} V\left(x_{1}+1, x_{2}\right) \\
& \left.+\left(1-\frac{\mu_{1} g_{1}\left(x_{1}, x_{2}\right)+\mu_{2} g_{2}\left(x_{1}, x_{2}\right)+\lambda}{R}\right) V\left(x_{1}, x_{2}\right)\right\}
\end{aligned}
$$

where $R$ is any fixed positive number. If we pick number $R$ such that

$$
R \geq \lambda+m \max \left(\mu_{1}, \mu_{2}\right),
$$


we can interpret Eq. (3) as the functional equation for a discrete-time Markov decision process whose transitions occur at fixed rate $R$ (i.e., the time unit between the transitions are $1 / R$, which is independent of the policy). This independence between transitions and the policy facilitates our analysis and allows us to use successive approximation, a well-known result in dynamic programming, to analyze Problem $(\mathrm{P})$.

Define for any $x_{1}, x_{2} \geq 0$, Markov policy $g$, and function $f: \bar{N}^{2} \mapsto \overline{R^{+}}$, the local cost function $c^{g}\left(x_{1}, x_{2}, f\right)$ by

$$
\begin{aligned}
c^{g}\left(x_{1}, x_{2}, f\right)= & \frac{1}{R}+\frac{\mu_{1} g_{1}\left(x_{1}, x_{2}\right)}{R}\left[p f\left(x_{1}-1, x_{2}+k\right)+(1-p) f\left(x_{1}-1, x_{2}\right)\right] \\
& +\frac{\mu_{2} g_{2}\left(x_{1}, x_{2}\right)}{R} f\left(x_{1}, x_{2}-1\right)+\frac{\lambda}{R} f\left(x_{1}+1, x_{2}\right) \\
& +\left(1-\frac{\mu_{1} g_{1}\left(x_{1}, x_{2}\right)+\mu_{2} g_{2}\left(x_{1}, x_{2}\right)+\lambda}{R}\right) f\left(x_{1}, x_{2}\right) .
\end{aligned}
$$

Thus, because of Eq. (3), the minimum expected makespan is the solution to the equation

$$
V\left(x_{1}, x_{2}\right)=\min _{g} c^{g}\left(x_{1}, x_{2}, V\right) .
$$

Construct the sequence of $\left\{V_{n}\left(x_{1}, x_{2}\right): n=0,1,2, \ldots\right\}$ as follows:

$$
\begin{aligned}
V_{0}\left(x_{1}, x_{2}\right) & =0 \quad \text { for all } x_{1}, x_{2}, \\
V_{n}(0,0) & =0 \quad \text { for all } n \geq 0, \\
V_{n}\left(x_{1}, x_{2}\right) & =\min _{g} c^{g}\left(x_{1}, x_{2}, V_{n-1}\right) \quad \text { for all } n \geq 1 .
\end{aligned}
$$

Lemma 1: Assume policy $\hat{g}=\left(\hat{g}_{1}\left(x_{1}, x_{2}\right), \hat{g}_{2}\left(x_{1}, x_{2}\right)\right)$ achieves the minimum in Eq. (9) for all $n$. Then, $\hat{g}$ is an optimal allocation policy for Problem $(P)$.

Proof: Lemma 1 is a special case of a general relationship between negative dynamic programming [10] and successive approximation first introduced by Strauch [12]. Note that since our action space is finite, Lemmas 3.1 and 9.1 in [12] provide the argument sufficient for establishing Lemma 1. For more detail on this proof, see [2].

Note that the set of Eqs. (7)-(9) are called the optimality equations for the $n$-stage problem. In the next subsection, we study this problem and its interpretation and prove that if $p \geq\left(\mu_{1}-\mu_{2}\right) / \mu_{1}$, policy $g^{*}$ is optimal for every $n \geq 1$.

\subsection{Solution to the $n$-Stage Problem}

Consider the sequence constructed by Eqs. (7)-(9). This sequence has the following interpretation. Consider the following finite horizon problem with $n$ transi- 
tions defined as horizon. Assume that in the queuing system in Figure 1, transitions occur at a fixed rate $R$, which is independent of the servicing policy. With probability $g_{i}\left(x_{1}, x_{2}\right)\left(\mu_{i} / R\right)$, the transition occurs in $Q_{i}$, with probability $\lambda / R$ that there is an arrival, and with probability $1-\left[\mu_{1} g_{1}\left(x_{1}, x_{2}\right)+\mu_{2} g_{2}\left(x_{1}, x_{2}\right)+\lambda\right] / R$ that there is a transition to the same state. Define the stopping time $\tau_{n}$ as the minimum of the makespan and horizon $n / R$. The objective is to minimize the expected value of $\tau_{n}$. It is easy to see that the optimality equation for this system coincides with Eqs. (7)-(9). This formulation is called the $n$-stage problem. This justifies why, in Section 2.2, Eqs. (7)-(9) are called the optimality equations for the $n$-stage problem. Naturally, if a policy achieves the minimum in Eq. (9) for a specific number $n$, the policy is called optimal at stage $n$. In fact, Lemma 1 shows the relationship between Problem (P) and the $n$-stage problem. In Lemma 1, we showed that solving Problem $(\mathrm{P})$ can be reduced to solving the $n$-stage problem. Hence, proving Theorem 1 is equivalent to proving the following theorem.

THEOREM 2: If $m \mu_{1} \mu_{2} / \lambda\left(\mu_{2}+k \mu_{1}\right)>1$ and $p \geq\left(\mu_{1}-\mu_{2}\right) / \mu_{1}$, then the policy that gives priority to $Q_{1}$ is optimal at every stage $n$.

Proof: The proof of this theorem requires a lengthy argument. For this reason, to clarify the ideas, we proceed as follows. First we present an outline of the proof where the main ideas are outlined. Then, we present all the details of the proof.

2.3. 1. Outline of the proof of Theorem 2. We prove Theorem 2 by induction on $n$, the number of stages. For that matter, we define the following conditions.

\section{Condition 1:}

$$
\frac{m \mu_{1} \mu_{2}}{\lambda\left(\mu_{2}+k \mu_{1}\right)}>1 .
$$

Condition 2:

$$
\begin{gathered}
p \geq \frac{\mu_{1}-\mu_{2}}{\mu_{1}} . \\
V_{n}^{1}\left(x_{1}, x_{2}\right)=\mu_{1}\left[p V_{n}\left(x_{1}-1, x_{2}+k\right)+(1-p) V_{n}\left(x_{1}-1, x_{2}\right)-V_{n}\left(x_{1}, x_{2}\right)\right] \\
V_{n}^{2}\left(x_{1}, x_{2}\right)=\mu_{2}\left[V_{n}\left(x_{1}, x_{2}-1\right)-V_{n}\left(x_{1}, x_{2}\right)\right] \\
\mathcal{D}_{n}\left(x_{1}, x_{2}\right)=V_{n}^{1}\left(x_{1}, x_{2}\right)-V_{n}^{2}\left(x_{1}, x_{2}\right), \\
\mathcal{F}_{n}\left(x_{1}, x_{2}\right)= \\
\quad p V_{n}\left(x_{1}-1, x_{2}+k-1\right)+(1-p) V_{n}\left(x_{1}-1, x_{2}\right) \\
-V_{n}\left(x_{1}, x_{2}-1\right), \\
\mathcal{G}_{n}\left(x_{1}, x_{2}\right)=
\end{gathered}
$$


Using the above expressions, we state the induction hypotheses for stage $n$ as follows:

$(\mathcal{H} 0)_{n}$ : policy $g^{*}$, which gives priority to $Q_{1}$, is optimal at stage $n$.

$(\mathcal{H} 1)_{n}: \mathcal{D}_{n}\left(x_{1}, x_{2}\right) \leq 0$ for every $x_{1} \geq 0, x_{2} \geq 0$.

$(\mathcal{H} 2)_{n}: \mathcal{F}_{n}\left(x_{1}, x_{2}\right) \leq 0$ for every $x_{1} \geq 0, x_{2} \geq 0$.

$(\mathcal{H} 3)_{n}: \mathcal{G}_{n}\left(x_{1}, x_{2}\right) \leq 0$ for every $x_{1} \geq 0, x_{2} \geq 0$.

The induction then proceeds as follows. First, we verify the validity of $(\mathcal{H} 0)_{1}-$ $(\mathcal{H} 3)_{1}$; this establishes the basis of the induction. For the induction step, we assume that $(\mathcal{H} 0)_{n}-(\mathcal{H} 3)_{n}$ are valid and prove that under this assumption and Condition 2, $(\mathcal{H} 0)_{n+1}-(\mathcal{H} 3)_{n+1}$ are true.

We note that $(\mathcal{H} 0)$ is sufficient to assert the validity of Theorem 2 . However, to prove inductively that $(\mathcal{H} 0)$ is true, we need $(\mathcal{H} 1)-(\mathcal{H} 3)$. Specifically, $(\mathcal{H} 0)_{n}-$ $(\mathcal{H} 3)_{n}$ are used to inductively establish Theorem 2 via the following lemmas:

LeMma 2: $(\mathcal{H} 0)_{n}$ and $(\mathcal{H} 1)_{n} \Rightarrow(\mathcal{H} 0)_{n+1}$.

LEMMA 3: $(\mathcal{H} 0)_{n+1}$ and $(\mathcal{H} 3)_{n} \Rightarrow(\mathcal{H} 3)_{n+1}$.

LeMma 4: $(\mathcal{H} 0)_{n+1},(\mathcal{H} 3)_{n}$, and $(\mathcal{H} 2)_{n} \Rightarrow(\mathcal{H} 2)_{n+1}$.

Lemma 5: $(\mathcal{H} 0)_{n+1},(\mathcal{H} 2)_{n}$, and $(\mathcal{H} 1)_{n} \Rightarrow(\mathcal{H} 1)_{n+1}$.

Condition 1 is sufficient to guarantee that the minimum expected makespan is finite.

2.3.2. Proof of Theorem 2. We proceed in two steps: First we establish the basis for the induction. Then, we complete the proof by establishing the induction step.

Basis for induction: From Eqs. (5), (7), and (9), we have $V_{1}\left(x_{1}, x_{2}\right)=$ $\min _{g}(1 / R)$. In this case, policy $g^{*}$ performs as well as any other policy at the first stage, so $(\mathcal{H} 0)_{1}$ is valid. As a result, $V_{1}\left(x_{1}, x_{2}\right)=1 / R$ for every $x_{1} \geq 0$, $x_{2} \geq 0$; hence, $\mathcal{D}_{1}\left(x_{1}, x_{2}\right)=\mathcal{F}_{1}\left(x_{1}, x_{2}\right)=\mathcal{G}_{1}\left(x_{1}, x_{2}\right)=0$, which proves the validity of $(\mathcal{H} 1)_{1}-(\mathcal{H} 3)_{1}$. The basis for the induction argument is now complete.

Induction step: As stated in Section 2.3.1, we assume that $(\mathcal{H} 0)_{n}-(\mathcal{H} 3)_{n}$ are valid and proceed to show that $(\mathcal{H} 0)_{n+1}-(\mathcal{H} 3)_{n+1}$ are also true via the proof of Lemmas 2-5. For the proof of Lemmas 2-5, we first establish the following facts.

Fact 1: For every $n \geq 1, x_{1} \geq 0$, and $x_{2} \geq 0$, the following relations hold:

$$
\begin{aligned}
& V_{n}\left(x_{1}+1, x_{2}\right) \geq V_{n}\left(x_{1}, x_{2}\right), \\
& V_{n}\left(x_{1}, x_{2}+1\right) \geq V_{n}\left(x_{1}, x_{2}\right) .
\end{aligned}
$$

Proof: As discussed earlier, $V_{n}\left(x_{1}, x_{2}\right)$ can be defined as the minimum of the expected value of the stopping time $\tau_{n}$ in the $n$-stage problem. For the $n$-stage 
problem, under any specific policy, and along every sample path of service completions, job interconnection, and job arrivals, the stopping time corresponding to the initial state $\left(x_{1}, x_{2}\right)$ is no longer than the stopping time corresponding to the initial state $\left(x_{1}+1, x_{2}\right)$ or to the initial state $\left(x_{1}, x_{2}+1\right)$. Therefore, the above inequalities hold.

Fact 2: For every $n \geq 1, x_{1} \geq 0$, and $x_{2} \geq 0$, the following relations hold:

$$
\begin{aligned}
& V_{n}^{1}\left(x_{1}, x_{2}\right) \leq 0, \\
& V_{n}^{2}\left(x_{1}, x_{2}\right) \leq 0 .
\end{aligned}
$$

Proof: In the $n$-stage problem, consider the effect of giving a small amount of processing time to a single job. Specifically, let $\delta \overline{V_{n}^{1}\left(x_{1}, x_{2}\right)}$ denote (to first order in $\delta$ ) the amount by which the expected value of $\tau_{n}$ would change from $V_{n}\left(x_{1}, x_{2}\right)$ if we were to give a job in $Q_{1}$ an extra $\delta$ amount of preprocessing time. Then,

$$
\begin{aligned}
\delta \overline{V_{n}^{1}\left(x_{1}, x_{2}\right)}= & \mu_{1} \delta\left(p V_{n}\left(x_{1}-1, x_{2}+k\right)+(1-p) V_{n}\left(x_{1}-1, x_{2}\right)\right) \\
& +\lambda \delta V_{n}\left(x_{1}+1, x_{2}\right)+\left(1-\mu_{1} \delta-\lambda \delta\right) V_{n}\left(x_{1}, x_{2}\right)-V_{n}\left(x_{1}, x_{2}\right) \\
= & \mu_{1} \delta\left(p V_{n}\left(x_{1}-1, x_{2}+k\right)+(1-p) V_{n}\left(x_{1}-1, x_{2}\right)-V_{n}\left(x_{1}, x_{2}\right)\right) \\
& +\lambda \delta\left(V_{n}\left(x_{1}+1, x_{2}\right)-V_{n}\left(x_{1}, x_{2}\right)\right) \\
\geq & \delta V_{n}^{1}\left(x_{1}, x_{2}\right)
\end{aligned}
$$

where the inequality holds because of Eq. (15).

On the other hand, since the processing times are exponential, we have

$$
\delta \overline{V_{n}^{1}\left(x_{1}, x_{2}\right)} \leq 0 \text { for all } x_{1}, x_{2},
$$

which results in Eq. (17).

Equation (18) can be proved in a similar way.

Note that the proof of this fact gives us a very intuitive interpretation of the functions $V_{n}^{1}\left(x_{1}, x_{2}\right), V_{n}^{2}\left(x_{1}, x_{2}\right)$, and $\mathcal{D}_{n}\left(x_{1}, x_{2}\right)$ for the $n$-stage problem. From the above discussion, it is clear that $V_{n}^{i}\left(x-1, x_{2}\right)$ can be viewed as the change in the expected value of $\tau_{n}$, in the absence of arrivals, as a result of giving a preprocessing to a job in $Q_{i}$. $\mathcal{D}_{n}\left(x_{1}, x_{2}\right)$ can be interpreted as the cost advantage of preprocessing a job in $Q_{2}$ over a job in $Q_{1}$, in the $n$-stage problem. Note that $\mathcal{D}_{n}\left(x_{1}, x_{2}\right)$ remains the same whether or not arrivals are included in the problem formulation. $(\mathcal{H} 1)_{n}$ implies that it is better to give the preprocessing to $Q_{1}$, which can be shown to be equivalent to the optimality of policy $g^{*}$.

Fact 3: Under Condition 2 (i.e., $\left.p \geq\left(\mu_{1}-\mu_{2}\right) / \mu_{1}\right)$,

$$
V_{n}^{1}\left(x_{1}, x_{2}-1\right)-V_{n}^{2}\left(x_{1}-1, x_{2}\right) \geq \mu_{1} \mathcal{G}_{n}\left(x_{1}, x_{2}\right) .
$$


PROOF:

$$
\begin{aligned}
& V_{n}^{1}\left(x_{1}, x_{2}-1\right)-V_{n}^{2}\left(x_{1}-1, x_{2}\right) \\
&= \mu_{1}\left[p V_{n}\left(x_{1}-1, x_{2}+k-1\right)+(1-p) V_{n}\left(x_{1}-1, x_{2}-1\right)-V_{n}\left(x_{1}, x_{2}-1\right)\right] \\
& \quad-\mu_{2}\left[V_{n}\left(x_{1}-1, x_{2}-1\right)-V_{n}\left(x_{1}-1, x_{2}\right)\right] \\
& \geq \mu_{1}\left[p V_{n}\left(x_{1}-1, x_{2}+k-1\right)+(1-p) V_{n}\left(x_{1}-1, x_{2}-1\right)-V_{n}\left(x_{1}, x_{2}-1\right)\right] \\
& \quad-\mu_{1}(1-p)\left[V_{n}\left(x_{1}-1, x_{2}-1\right)-V_{n}\left(x_{1}-1, x_{2}\right)\right] \\
&= \mu_{1}\left\{p\left[V_{n}\left(x_{1}-1, x_{2}+k-1\right)-V_{n}\left(x_{1}-1, x_{2}\right)\right]\right. \\
&\left.\quad+\left[V_{n}\left(x_{1}-1, x_{2}\right)-V_{n}\left(x_{1}, x_{2}-1\right)\right]\right\} \\
& \geq \mu_{1} \mathcal{G}_{n}\left(x_{1}, x_{2}\right) .
\end{aligned}
$$

The first inequality is a result of Condition 2 and the second inequality follows from Eq. (16) and $k \geq 1$. Intuitively, $\mathcal{G}_{n}\left(x_{1}, x_{2}\right)$ can be interpreted as follows. Consider the $n$-stage problem where a new job is added to the system and one has the option of assigning it to either $Q_{1}$ or $Q_{2}$. Then, $\mathcal{G}_{n}\left(x_{1}, x_{2}\right)$ gives the cost advantage of assigning the extra job to $Q_{1}$ instead of $Q_{2} \cdot(\mathcal{H} 3)_{n}$ implies that at every stage $n$, it is better to assign the extra job to $Q_{1}$.

Fact 4: Under Condition 2 (i.e., $\left.p \geq\left(\mu_{1}-\mu_{2}\right) / \mu_{1}\right)$,

$$
\begin{aligned}
& V_{n}^{1}\left(x_{1}, x_{2}-1\right)-p V_{n}^{2}\left(x_{1}-1, x_{2}+k-1\right)-(1-p) V_{n}^{2}\left(x_{1}-1, x_{2}\right) \\
& \geq \mu_{1}\left\{-(1-p) \mathcal{G}_{n}\left(x_{1}, x_{2}-1\right)-p \mathcal{G}_{n}\left(x_{1}, x_{2}\right)-(1-p) \mathcal{F}_{n}\left(x_{1}, x_{2}-1\right)\right. \\
& \left.\quad+(1-p) \mathcal{F}_{n}\left(x_{1}, x_{2}\right)\right\} .
\end{aligned}
$$

Proof:

$$
\begin{aligned}
V_{n}^{1}\left(x_{1}, x_{2}-1\right)-p V_{n}^{2}\left(x_{1}-1, x_{2}+k-1\right)-(1-p) V_{n}^{2}\left(x_{1}-1, x_{2}\right) \\
=\mu_{1}\left[p V_{n}\left(x_{1}-1, x_{2}+k-1\right)+(1-p) V_{n}\left(x_{1}-1, x_{2}-1\right)-V\left(x_{1}-1, x_{2}\right)\right] \\
-\mu_{2}\left[p V_{n}\left(x_{1}-1, x_{2}+k-2\right)-p V_{n}\left(x_{1}-1, x_{2}+k-1\right)\right. \\
\left.\quad+(1-p) V_{n}\left(x_{1}-1, x_{2}-1\right)-(1-p) V_{n}\left(x_{1}-1, x_{2}\right)\right] \\
\geq \mu_{1}\left[p V_{n}\left(x_{1}-1, x_{2}+k-1\right)+(1-p) V_{n}\left(x_{1}-1, x_{2}-1\right)-V\left(x_{1}-1, x_{2}\right)\right] \\
-\mu_{1}(1-p)\left[p V_{n}\left(x_{1}-1, x_{2}+k-2\right)-p V_{n}\left(x_{1}-1, x_{2}+k-1\right)\right. \\
\left.\quad+(1-p) V_{n}\left(x_{1}-1, x_{2}-1\right)-(1-p) V_{n}\left(x_{1}-1, x_{2}\right)\right] \\
=\mu_{1}\left\{p\left[V_{n}\left(x_{1}-1, x_{2}+k-1\right)-V_{n}\left(x_{1}, x_{2}-1\right)\right]+(1-p) V_{n}\left(x_{1}-1, x_{2}-1\right)\right. \\
\quad-(1-p)\left[V_{n}\left(x_{1}, x_{2}-1\right)-p V_{n}\left(x_{1}-1, x_{2}+k-1\right)\right. \\
\left.\quad-(1-p) V_{n}\left(x_{1}-1, x_{2}\right)\right] \\
\left.\quad+(1-p)\left[-p V_{n}\left(x_{1}-1, x_{2}+k-2\right)-(1-p) V_{n}\left(x_{1}-1, x_{2}-1\right)\right]\right\} \\
\geq \mu_{1}\left\{-p \mathcal{G}_{n}\left(x_{1}, x_{2}\right)+(1-p) V_{n}\left(x_{1}-1, x_{2}-1\right)\right. \\
\left.\quad+(1-p) \mathcal{F}_{n}\left(x_{1}, x_{2}\right)-(1-p)\left[\mathcal{F}_{n}\left(x_{1}, x_{2}-1\right)+V_{n}\left(x_{1}, x_{2}-2\right)\right]\right\} \\
=\mu_{1}\left\{-p \mathcal{G}_{n}\left(x_{1}, x_{2}\right)+(1-p)\left[V_{n}\left(x_{1}-1, x_{2}-1\right)-V_{n}\left(x_{1}, x_{2}-2\right)\right]\right. \\
\left.\quad+(1-p) \mathcal{F}_{n}\left(x_{1}, x_{2}\right)-(1-p) \mathcal{F}_{n}\left(x_{1}, x_{2}-1\right)\right\} \\
=\mu_{1}\left\{-p \mathcal{G}_{n}\left(x_{1}, x_{2}\right)-(1-p) \mathcal{G}_{n}\left(x_{1}, x_{2}-1\right)\right. \\
\left.\quad+(1-p) \mathcal{F}_{n}\left(x_{1}, x_{2}\right)-(1-p) \mathcal{F}_{n}\left(x_{1}, x_{2}-1\right)\right\} .
\end{aligned}
$$


The first inequality is a result of Condition 2 and the second inequality follows from Eq. (16), where $k \geq 1$, and Eq. (14).

Based on Facts 1-4, we prove Lemmas 2-5, which are needed for the completion of the induction step.

Lemma 2: Assume Conditions 1 and 2 hold. If $\mathcal{D}_{n}\left(x_{1}, x_{2}\right) \leq 0$ for all $x_{1} \geq 0, x_{2} \geq 0$, then $g^{*}$ is optimal at stage $n+1$.

Proof: By the definition of $g^{*}$, which gives priority to $Q_{1}$, we know that for any arbitrary policy $g$,

$$
g_{1}\left(x_{1}, x_{2}\right) \leq g_{1}^{*}\left(x_{1}, x_{2}\right) \text { for all } x_{1}, x_{2} .
$$

Without loss of generality, we have restricted our attention to policies for which

$$
g_{1}\left(x_{1}, x_{2}\right)+g_{2}\left(x_{1}, x_{2}\right)=g_{1}^{*}\left(x_{1}, x_{2}\right)+g_{2}^{*}\left(x_{1}, x_{2}\right)=m .
$$

If $x_{1}+x_{2} \leq m, g^{*}$, by its definition, utilizes $x_{1}+x_{2}$ servers; therefore, it results in an optimal action. When $x_{1}+x_{2}>m$, we prove for any policy $g$ that $g_{1}\left(x_{1}, x_{2}\right)<$ $g_{1}^{*}\left(x_{1}, x_{2}\right)=\min \left(m, x_{1}\right)$ can be imposed at stage $n+1$ by reallocating one server from a job in $Q_{2}$ to a job $Q_{1}$. Repetition of the same argument shows that $g^{*}$ is optimal at stage $n+1$. Therefore, to complete the proof, we compare, at stage $n+1$, the local cost functions due to policy $g^{I}$ and $g$, where

$$
g_{1}^{I}\left(x_{1}, x_{2}\right)=g_{1}\left(x_{1}, x_{2}\right)+1 \quad \text { and } \quad g_{2}^{I}\left(x_{1}, x_{2}\right)=g_{2}\left(x_{1}, x_{2}\right)-1 .
$$

We have

$$
\begin{aligned}
c^{g^{I}}\left(x_{1}, x_{2}, V_{n}\right)-c^{g}\left(x_{1}, x_{2}, V_{n}\right) \\
=\frac{\mu_{1}}{R}\left(p V_{n}\left(x_{1}-1, x_{2}+k\right)+(1-p) V_{n}\left(x_{1}-1, x_{2}\right)\right) \\
\quad-\frac{\mu_{2}}{R} V_{n}\left(x_{1}, x_{2}-1\right)-\frac{\mu_{1}}{R} V_{n}\left(x_{1}, x_{2}\right)+\frac{\mu_{2}}{R} V_{n}\left(x_{1}, x_{2}\right) \\
=\frac{\mu_{1}}{R}\left(p V_{n}\left(x_{1}-1, x_{2}+k\right)+(1-p) V_{n}\left(x_{1}-1, x_{2}\right)-V_{n}\left(x_{1}, x_{2}\right)\right) \\
\quad-\frac{\mu_{2}}{R}\left(V_{n}\left(x_{1}, x_{2}-1\right)-V n n\right) \\
=\frac{1}{R} \mathcal{D}_{n}\left(x_{1}, x_{2}\right) \leq 0 .
\end{aligned}
$$

The inequality in Eq. (21) together with argument proceeding it competes the proof of Lemma 2.

Lemma 3: Assume that Conditions 1 and 2 hold. If $g^{*}$ is optimal at stage $n+1$ and $\mathcal{G}_{n}\left(x_{1}, x_{2}\right) \leq 0$ for all $x_{1}, x_{2}$, then $\mathcal{G}_{n+1}\left(x_{1}, x_{2}\right) \leq 0$. 
Proof: In Appendix A, we show the following:

(i) If $x_{1}>m$,

$$
\begin{aligned}
\mathcal{G}_{n+1}\left(x_{1}, x_{2}\right)= & \frac{\mu_{1} m}{R}\left[p \mathcal{G}_{n}\left(x_{1}-1, x_{2}+k\right)+(1-p) \mathcal{G}_{n}\left(x_{1}-1, x_{2}\right)\right] \\
& +\frac{\lambda}{R} \mathcal{G}_{n}\left(x_{1}+1, x_{2}\right)+\left(1-\frac{\mu_{1} m+\lambda}{R}\right) \mathcal{G}_{n}\left(x_{1}, x_{2}\right) .
\end{aligned}
$$

(ii) If $x_{1} \leq m<x_{1}+x_{2}$.

$$
\begin{aligned}
\mathcal{G}_{n+1}\left(x_{1}, x_{2}\right) \leq & \frac{\mu_{1}\left(x_{1}-1\right)}{R}\left[p \mathcal{G}_{n}\left(x_{1}-1, x_{2}+k\right)+(1-p) \mathcal{G}_{n}\left(x_{1}-1, x_{2}\right)\right] \\
& +\frac{\mu_{2}\left(m-x_{1}\right)}{R} V_{n}\left(x_{1}, x_{2}-1\right)+\frac{\lambda}{R} \mathcal{G}_{n}\left(x_{1}+1, x_{2}\right) \\
& +\left(1-\frac{\mu_{1} x_{1}+\mu_{2}\left(m-x_{1}\right)+\lambda}{R}\right) \mathcal{G}_{n}\left(x_{1}, x_{2}\right)
\end{aligned}
$$

(iii) If $x_{1}+x_{2} \leq m$,

$$
\begin{aligned}
\mathcal{G}_{n+1}\left(x_{1}, x_{2}\right) \leq & \frac{\mu_{1}\left(x_{1}-1\right)}{R}\left[p \mathcal{G}_{n}\left(x_{1}-1, x_{2}+k\right)+(1-p) \mathcal{G}_{n}\left(x_{1}-1, x_{2}\right)\right] \\
& +\frac{\mu_{2}\left(x_{2}-1\right)}{R} \mathcal{G}_{n}\left(x_{1}, x_{2}-1\right)+\frac{\lambda}{R} \mathcal{G}_{n}\left(x_{1}+1, x_{2}\right) \\
& +\left(1-\frac{\mu_{1} x_{1}+\mu_{2}\left(x_{2}-1\right)+\lambda}{R}\right) \mathcal{G}_{n}\left(x_{1}, x_{2}\right)
\end{aligned}
$$

Note that using Eq. (4) and the induction hypothesis, namely $\mathcal{G}_{n}\left(x_{1}, x_{2}\right) \leq 0$ for all $x_{1}, x_{2}$, we can see that the left-hand sides in Eqs. (22)-(24) are nonpositive; that is,

$$
\mathcal{G}_{n+1}\left(x_{1}, x_{2}\right) \leq 0 \text {. }
$$

Lemma 4: Assume that Conditions 1 and 2 hold. If $g^{*}$ is optimal at stage $n+1$, $\mathcal{G}_{n}\left(x_{1}, x_{2}\right) \leq 0$, and $\mathcal{F}_{n}\left(x_{1}, x_{2}\right) \leq 0$ for all $x_{1}, x_{2}$, then $\mathcal{F}_{n+1}\left(x_{1}, x_{2}\right) \leq 0$.

Proof: In Appendix A, we show the following:

(i) If $x_{1}>m$,

$$
\begin{aligned}
\mathcal{F}_{n+1}\left(x_{1}, x_{2}\right)= & \frac{\mu_{1} m}{R}\left[p \mathcal{F}_{n}\left(x_{1}-1, x_{2}+k\right)+(1-p) \mathcal{F}_{n}\left(x_{1}-1, x_{2}\right)\right] \\
& +\frac{\lambda}{R} \mathcal{F}_{n}\left(x_{1}+1, x_{2}\right)+\left(1-\frac{\mu_{1} m+\lambda}{R}\right) \mathcal{F}_{n}\left(x_{1}, x_{2}\right) .
\end{aligned}
$$


(ii) If $x_{1} \leq m<x_{1}+x_{2}$,

$$
\begin{aligned}
\mathcal{F}_{n+1}\left(x_{1}, x_{2}\right) \leq & \frac{\mu_{1}\left(x_{1}-1\right)}{R}\left[p \mathcal{F}_{n}\left(x_{1}-1, x_{2}+k\right)+(1-p) \mathcal{F}_{n}\left(x_{1}-1, x_{2}\right)\right] \\
& +\frac{\mu_{2}\left(m-x_{1}\right)}{R} \mathcal{F}_{n}\left(x_{1}, x_{2}-1\right)+\frac{\lambda}{R} \mathcal{F}_{n}\left(x_{1}+1, x_{2}\right) \\
& +\left(1-\frac{\mu_{1}\left(x_{1}-1\right)+\mu_{2}\left(m-x_{1}\right)+\lambda}{R}\right) \mathcal{F}_{n}\left(x_{1}, x_{2}\right) \\
& +\frac{\mu_{1}}{R}\left\{(1-p) \mathcal{G}_{n}\left(x_{1}, x_{2}-1\right)+p \mathcal{G}_{n}\left(x_{1}, x_{2}\right)\right. \\
& \left.+(1-p) \mathcal{F}_{n}\left(x_{1}, x_{2}-1\right)\right\} .
\end{aligned}
$$

(iii) If $x_{1}+x_{2} \leq m$,

$$
\begin{aligned}
\mathcal{F}_{n+1}\left(x_{1}, x_{2}\right) \leq & \frac{\mu_{1}\left(x_{1}-1\right)}{R}\left[p \mathcal{F}_{n}\left(x_{1}-1, x_{2}+k\right)+(1-p) \mathcal{F}_{n}\left(x_{1}-1, x_{2}\right)\right] \\
& +\frac{\mu_{2}\left(x_{2}-1\right)}{R} \mathcal{F}_{n}\left(x_{1}, x_{2}-1\right)+\frac{\lambda}{R} \mathcal{F}_{n}\left(x_{1}+1, x_{2}\right) \\
& +\left(1-\frac{\mu_{1}\left(x_{1}-1\right)+\mu_{2}\left(x_{2}-1\right)+\lambda}{R}\right) \mathcal{F}_{n}\left(x_{1}, x_{2}\right) \\
& +\frac{\mu_{1}}{R}\left\{(1-p) \mathcal{G}_{n}\left(x_{1}, x_{2}-1\right)+p \mathcal{G}_{n}\left(x_{1}, x_{2}\right)\right. \\
& \left.\quad+(1-p) \mathcal{F}_{n}\left(x_{1}, x_{2}-1\right)\right\} .
\end{aligned}
$$

Note that, again, the right-hand sides in Eqs. (25)-(27) are nonpositive, because of Eq. (4) and the assumption that $\mathcal{F}_{n}\left(x_{1}, x_{2}\right) \leq 0$ for all $x_{1}, x_{2}$; that is,

$$
\mathcal{F}_{n+1}\left(x_{1}, x_{2}\right) \leq 0 \text {. }
$$

Lemma 5: Assume that Conditions 1 and 2 hold. If $g^{*}$ is optimal at stage $n+1$, $\mathcal{F}_{n}\left(x_{1}, x_{2}\right) \leq 0$, and $\mathcal{D}_{n}\left(x_{1}, x_{2}\right) \leq 0$ for all $x_{1}, x_{2}$, then $\mathcal{D}_{n+1}\left(x_{1}, x_{2}\right) \leq 0$.

Proof: In Appendix A, we show the following:

(i) If $x_{1}>m$,

$$
\begin{aligned}
\mathcal{D}_{n+1}\left(x_{1}, x_{2}\right)= & \frac{\mu_{1} m}{R}\left[p \mathcal{D}_{n}\left(x_{1}-1, x_{2}+k\right)+(1-p) \mathcal{D}_{n}\left(x_{1}-1, x_{2}\right)\right] \\
& +\frac{\lambda}{R} \mathcal{D}_{n}\left(x_{1}+1, x_{2}\right)+\left(1-\frac{\mu_{1} m+\lambda}{R}\right) \mathcal{D}_{n}\left(x_{1}, x_{2}\right) .
\end{aligned}
$$


(ii) If $x_{1} \leq m<x_{1}+x_{2}$,

$$
\begin{aligned}
\mathcal{D}_{n+1}\left(x_{1}, x_{2}\right)= & \frac{\mu_{1}\left(x_{1}-1\right)}{R}\left[p \mathcal{D}_{n}\left(x_{1}-1, x_{2}+k\right)\right. \\
& \left.+(1-p) \mathcal{D}_{n}\left(x_{1}-1, x_{2}\right)\right] \\
& +\frac{\mu_{2}\left(m-x_{1}\right)}{R} \mathcal{D}_{n}\left(x_{1}, x_{2}-1\right)+\frac{\lambda}{R} \mathcal{D}_{n}\left(x_{1}+1, x_{2}\right) \\
& +\left(1-\frac{\mu_{1} x_{1}+\mu_{2}\left(m-x_{1}\right)+\lambda}{R}\right) \mathcal{D}_{n}\left(x_{1}, x_{2}\right)
\end{aligned}
$$

(iii) If $x_{1}+x_{2} \leq m$,

$$
\begin{aligned}
\mathcal{D}_{n+1}\left(x_{1}, x_{2}\right) \leq & \frac{\mu_{1}\left(x_{1}-1\right)}{R}\left[p \mathcal{D}_{n}\left(x_{1}-1, x_{2}+k\right)\right. \\
& \left.+(1-p) \mathcal{D}_{n}\left(x_{1}-1, x_{2}\right)\right] \\
& +\frac{\mu_{2}\left(x_{2}-1\right)}{R} \mathcal{D}_{n}\left(x_{1}, x_{2}-1\right)+\frac{\lambda}{R} \mathcal{D}_{n}\left(x_{1}+1, x_{2}\right) \\
& +\left(1-\frac{\mu_{1} x_{1}+\mu_{2} x_{2}+\lambda}{R}\right) \mathcal{D}_{n}\left(x_{1}, x_{2}\right) \\
& +\frac{\mu_{1} \mu_{2}}{R} \mathcal{F}_{n}\left(x_{1}, x_{2}\right) .
\end{aligned}
$$

Because of Eq. (4) and the induction hypothesis on $\mathcal{D}_{n}\left(x_{1}, x_{2}\right)$ and $\mathcal{F}_{n}\left(x_{1}, x_{2}\right)$ [viz. for all $x_{1}$ and $x_{2}, \mathcal{D}_{n}\left(x_{1}, x_{2}\right) \leq 0$ and $\mathcal{F}_{n}\left(x_{1}, x_{2}\right) \leq 0$ ], we know that the righthand side of Eqs. (28)-(30) are nonpositive; that is, for all $x_{1}, x_{2}$,

$$
\mathcal{D}_{n+1}\left(x_{1}, x_{2}\right) \leq 0 \text {. }
$$

The induction step is now complete, as $(\mathcal{H} 0)_{n+1}-(\mathcal{H} 3)_{n+1}$ are true because of Lemmas 2, 5, 4, and 3, respectively. This concludes the proof of Theorem 2.

\section{DISCUSSION}

In this section, we first present an example that illustrates the role of the condition expressed by Eq. (1) in Theorem 1. The example shows that Eq. (1) is sufficient but not necessary to ensure the optimality of policy $g^{*}$ that gives priority to $Q_{1}$. Furthermore, the example shows that when the condition expressed to Eq. (1) is not satisfied, then, depending on $\mu_{1}, \mu_{2}$, and $p$, an optimal allocation policy either gives priority to $Q_{1}$, or to $Q_{2}$, or is state dependent. We identify an instance where it is possible to establish the optimality of the policy that gives priority to $Q_{2}$ for Problem (P) when Eq. (1) is not satisfied. We discuss this instance after Example 3.1.

Example 3.1: Consider the system in Figure 1. Let the initial state be $\left(x_{1}, x_{2}\right)=$ $(2,2)$. Suppose there are three machines available (i.e., $m=3)$ and each job moving 
from $Q_{1}$ to $Q_{2}$ creates two jobs in $Q_{2}$ (i.e., $k=2$ ). Let $\mu_{1}=2 \mu_{2}$ and $\lambda=0$. Hence, the requirement expressed by Eq. (1) is

$$
p \geq \frac{1}{2} .
$$

As discussed earlier, we only need to restrict attention to nonidling allocation strategies. Thus, when the state $\left(x_{1}, x_{2}\right)$ is such that $x_{1}+x_{2} \leq m$, the allocation decision is trivial. For our example, there are only three states for which the allocation is not trivial: when the system is at the initial state $(2,2)$ and if the system enters states $(1,4)$ or $(1,3)$. We consider two nonidling priority allocation strategies: policy $g^{*}$ that gives priority to $Q_{1}$ and policy $g$ that gives priority to $Q_{2}$. These two policies differ from each other in their allocation of servers if the system is in states $(2,2)$, $(1,4)$, and $(1,3)$ (for all other states, both policies act in the same way).

Define the functions $V^{g^{*}}$ and $V^{g}$ as the expected makespan under policy $g^{*}$ and policy $g$, respectively. At each state $\left(x_{1}, x_{2}\right)$, the function $V$ is defined as the minimum expected makespan. Then,

$$
\begin{aligned}
& V^{g^{*}}(0,1)=V^{g}(0,1)=\frac{1}{\mu_{2}}=\frac{2}{\mu_{1}}, \\
& V^{g^{*}}(0,2)=V^{g}(0,2)=\frac{3}{2 \mu_{2}}=\frac{3}{\mu_{1}}, \\
& V^{g^{*}}(0,3)=V^{g}(0,3)=\frac{11}{6 \mu_{2}}=\frac{11}{3 \mu_{1}}, \\
& V^{g^{*}}(0,4)=V^{g}(0,4)=\frac{13}{6 \mu_{2}}=\frac{13}{3 \mu_{1}}, \\
& V^{g^{*}}(0,5)=V^{g}(0,5)=\frac{15}{6 \mu_{2}}=\frac{15}{3 \mu_{1}}, \\
& V^{g^{*}}(1,0)=V^{g}(1,0)=\frac{1}{\mu_{1}}+p V(0,2)=\frac{3 p+1}{\mu_{1}}, \\
& V^{g^{*}}(1,1)=V^{g}(1,1) \\
& =\frac{1}{\mu_{1}+\mu_{2}}+\frac{\mu_{1}}{\mu_{1}+\mu_{2}}[p V(0,3)+(1-p) V(0,1)]+\frac{\mu_{2}}{\mu_{1}+\mu_{2}} V(1,0) \\
& =\frac{19 p+21}{9 \mu_{1}} \\
& V^{g^{*}}(1,2)=V^{g}(1,2) \\
& =\frac{1}{\mu_{1}+2 \mu_{2}}+\frac{\mu_{1}}{\mu_{1}+2 \mu_{2}}[p V(0,4)+(1-p) V(0,2)]+\frac{2 \mu_{2}}{\mu_{1}+2 \mu_{2}} V(1,1) \\
& =\frac{31 p+57}{18 \mu_{1}} \text {. }
\end{aligned}
$$


At states $(1,3),(1,4)$, and $(2,2)$, the two strategies $g^{*}$ and $g$ allocate servers differently. Thus, we have

$$
\begin{aligned}
& V^{g^{*}}(1,3)=\frac{1}{\mu_{1}+2 \mu_{2}}+\frac{\mu_{1}}{\mu_{1}+2 \mu_{2}}[p V(0,5)+(1-p) V(0,3)]+\frac{2 \mu_{2}}{\mu_{1}+2 \mu_{2}} V(1,2), \\
& =\frac{55 p+141}{36 \mu_{1}} \text {, } \\
& V^{g}(1,3)=\frac{1}{3 \mu_{2}}+V(1,2)=\frac{31 p+69}{36 \mu_{2}}, \\
& V^{g^{*}}(1,4)=\frac{1}{\mu_{1}+2 \mu_{2}}+\frac{\mu_{1}}{\mu_{1}+2 \mu_{2}}[p V(0,6)+(1-p) V(0,4)] \\
& +\frac{2 \mu_{2}}{\mu_{1}+2 \mu_{2}} V(1,3) \\
& V^{g}(1,4)=\frac{1}{3 \mu_{2}}+V(1,3) \text {, } \\
& V(2,1)=\frac{1}{2 \mu_{1}+\mu_{2}}+\frac{2 \mu_{1}}{2 \mu_{1}+\mu_{2}}[p V(1,3)+(1-p) V(1,1)] \\
& +\frac{\mu_{2}}{2 \mu_{1}+\mu_{2}} V(1,2) \\
& V^{g^{*}}(2,2)=\frac{1}{2 \mu_{1}+\mu_{2}}+\frac{2 \mu_{1}}{2 \mu_{1}+\mu_{2}}[p V(1,4)+(1-p) V(1,2)] \\
& +\frac{\mu_{2}}{2 \mu_{1}+\mu_{2}} V(2,1) \\
& V^{g}(2,2)=\frac{1}{\mu_{1}+2 \mu_{2}}+\frac{\mu_{1}}{\mu_{1}+2 \mu_{2}}[p V(1,4)+(1-p) V(1,2)] \\
& +\frac{2 \mu_{2}}{\mu_{1}+2 \mu_{2}} V(2,1)
\end{aligned}
$$

where, as mentioned earlier, at each state $\left(x_{1}, x_{2}\right), V\left(x_{1}, x_{2}\right)$ is defined as

$$
V\left(x_{1}, x_{2}\right)=\min \left\{V^{g^{*}}\left(x_{1}, x_{2}\right), V^{g}\left(x_{1}, x_{2}\right)\right\} .
$$

To determine $V\left(x_{1}, x_{2}\right)$, we first look at the expected makespan under strategies $g$ and $g^{*}$ if the system is at either states $(1,3)$ or $(1,4)$. By comparison of these strategies, we identify the least expected makespan for different values of $p$. We get

$$
V(1,3)= \begin{cases}V^{g^{*}}(1,3) & \text { if } p \geq 0.4285 \\ V^{g}(1,3) & \text { otherwise }\end{cases}
$$


and

$$
V(1,4)= \begin{cases}V^{g^{*}}(1,4) & \text { if } p \geq 0.4285 \\ V^{g}(1,4) & \text { otherwise }\end{cases}
$$

We use the above relations to determine $V(2,2)$. We first assume $p \geq 0.4285$. Using Eqs. (39) and (40), we obtain

$$
V(2,2)=V^{g^{*}}(2,2) \quad \text { if } p \geq 0.4285 \text {. }
$$

Next, we assume $p \leq 0.4285$. Using Eqs. (39) and (40), we get

$$
V(2,2)= \begin{cases}V^{g}(2,2) & \text { if } p \leq 0.4267 \\ V^{g^{*}}(2,2) & \text { if } 0.4267 \leq p \leq 0.4285\end{cases}
$$

From the above, we conclude the following:

1. If $p \in[0,0.4267]$, it is always optimal to follow policy $g$.

2. If $p \in[0.4267,0.4285]$, the optimal policy depends on the state; at states $(1,3)$ and $(1,4)$, it is optimal to follow $g$, whereas at $(2,2)$, the optimal allocation coincides with policy $g^{*}$.

3. If $p \in[0.4285,1]$, policy $g^{*}$ is optimal.

Comparing Inequality (31) with the above result, we conclude the following: (i) If the requirement expressed by Eq. (1) is not satisfied, the policy $g^{*}$ is not, in general, optimal. (ii) The requirement expressed by Eq. (1) is only sufficient but not necessary to guarantee the optimality of policy $g^{*}$. (iii) The optimal policy need not be a priority policy (i.e., an optimal allocation may depend on the state of the system).

Special Case: $k=1$ : As mentioned at the beginning of this section and demonstrated in Example 3.1, if Eq. (1) is not satisfied, the result of this article provides no conclusive evidence about the form of an optimal allocation policy. However, it is possible to determine an optimal allocation strategy for Problem (P) when Eq. (1) is not true and $k=1$. Such a strategy is desired in the following theorem.

Theorem 3: Assume $m \mu_{1} \mu_{2} / \lambda\left(\mu_{2}+k \mu_{1}\right)>1$ and $p \leq\left(\mu_{1}-\mu_{2}\right) / \mu_{1}$. Under these conditions, Policy $\tilde{g}$, which gives priority to $Q_{2}$, is an optimal policy for Problem $(P)$ when $k=1$.

Theorem 3 can be proved by arguments similar to those used to prove Theorem 1. These arguments are presented in Appendix B.

Theorems 1 and 3 show that it is possible to determine an optimal allocation policy for any combination of $\mu_{1}, \mu_{2}$, and $p$ when each job interconnecting from $Q_{1}$ to $Q_{2}$ creates exactly one job in $Q_{2}$.

\section{CONCLUSION}

The main result of the article provides a condition sufficient to guarantee the optimality of the policy that gives priority to $Q_{1}$ for Problem $(\mathrm{P})$. In this case, where 
every task interconnecting from $Q_{1}$ to $Q_{2}$ creates exactly one job in $Q_{2}$ (i.e., $k=1$ ), we have determined an optimal allocation policy for any combination of values of $\mu_{1}, \mu_{2}$, and $p$.

Determining an optimal allocation policy for any combination of values of $\mu_{1}, \mu_{2}$, and $p$ when $k>1$ is a problem worthy of investigation. Another situation similar to Problem $(\mathrm{P})$ that is practically significant arises when the number of processors required to process a job at a certain queue depends on the queue. Determining an optimal allocation strategy for such a class of problems appears to be a challenging task. The determination of an optimal allocation strategy that stochastically minimizes makespan for each of the above problems is also an interesting problem worthy of investigation.

\section{Acknowledgments}

This research was supported in part by AFOSR grant F49620-96-1-0028 and ARO grant DAAHO4-96-1-0377.

\section{References}

1. Bruno, J., Downey, P., \& Frederickson, G.N. (1981). Scheduling tasks with exponential service times to minimize the expected flow time and makespan. Journal of the Association of Computational Mathematics 28.

2. Javidi, T., Song, N., \& Teneketzis, D. (2000). Expected makespan minimization on identical machines in two interconnected queues. University of Michigan Control Group Report CGR-00-13, Ann Arbor, MI.

3. Liu, Z. (1995). Scheduling of random task graphs on parallel processors. IEEE Proceeding of the 3rd MASCOT, Workshop on Modeling and Analysis of Simulation of Computer and Telecommunication Systems, pp. 143-147.

4. Liu, Z. \& Righter, R. (1997). Optimal scheduling on parallel processors with precedence constraints and general cost. Probability in the Engineering and Informational Sciences 11: 79-93.

5. Liu, Z. \& Righter, R. (2001). Optimal parallel processing of random task graphs. Journal of Scheduling (to appear).

6. Liu, Z. \& Sanlaville, E. (1995). Preemptive scheduling with variable profile, precedence constraints and due dates. Discrete Applied Mathematics 58: 253-280.

7. Liu, Z. \& Sanlaville, E. (1995). Profile scheduling by list algorithms. In P. Chretienne, E.G. Coffmann Jr., J.K. Lenstra, \& Z. Liu (eds.), Scheduling theory and its applications. New York: John Wiley, pp. 95-114.

8. Pinedo, M. (1995). Scheduling. Englewood Cliffs, NJ: Prentice-Hall.

9. Pinedo, M. \& Schrage, L. (1982). Stochastic shop scheduling: A survey. In M. Dempster, J.K. Lenstra, \& A.H.G. Rinnooy Kan (eds.), Deterministic and stochastic scheduling. Dordrecht: D. Reidel.

10. Ross, S. (1983). Introduction to stochastic dynamic programming. New York: Academic Press.

11. Song, N. \& Teneketzis, D. (1998). Makespan minimization on identical parallel machines in two interconnected queues. University of Michigan Control Group Report CGR-98-15, Ann Arbor, MI.

12. Strauch, R.E. (1966). Negative dynamic programming. Annals of Mathematical Statistics 37: 871-890.

13. Weber, R.R. (1979). Optimal organization of multiserver systems. [Unpublished] Ph.D. thesis, University of Cambridge, Cambridge.

14. Weber, R.R. (1982). Scheduling jobs with stochastic processing requirements on parallel machine to minimize makespan or flowtime. Journal of Applied Probability 19: 167-182.

15. Weiss, G. (1982). Multiserver stochastic scheduling. In M. Dempster et al. (eds.), Deterministic and stochastic scheduling. Dordrecht: D. Reidel.

16. Whitt, W. (1972). Embedded renewal process in the gi/g/s queue. Journal of Applied Probability 9: 650-658. 
17. Wittle, P. (1982). Optimization over time. New York: Wiley.

18. Van Der Heyden, L. (1981). Scheduling jobs with exponential processing and arrival times on identical processors so as to minimize the expected makespan. Mathematics of Operations Research 6(2): 305-312.

\section{APPENDIX A \\ Proofs of Equations (22)-(30)}

To prove Eqs. (22)-(30), we incorporate the assumption that $g^{*}$ is optimal at stage $n+1$, which translates to

$$
V_{n+1}\left(x_{1}, x_{2}\right)=c^{g^{*}}\left(x_{1}, x_{2}, V_{n}\right) .
$$

Proof of Equation (22): If $x_{1}>m$,

$$
\begin{array}{ll}
g_{1}^{*}\left(x_{1}-1, x_{2}\right)=m, & g_{2}^{*}\left(x_{1}-1, x_{2}\right)=0, \\
g_{1}^{*}\left(x_{1}, x_{2}-1\right)=m, & g_{2}^{*}\left(x_{1}, x_{2}-1\right)=0 .
\end{array}
$$

So we have

$$
\begin{aligned}
\mathcal{G}_{n+1}\left(x_{1}, x_{2}\right)= & V_{n+1}\left(x_{1}-1, x_{2}\right)-V_{n+1}\left(x_{1}, x_{2}-1\right) \\
= & \frac{\mu_{1} m}{R}\left[p V_{n}\left(x_{1}-2, x_{2}+k\right)+(1-p) V_{n}\left(x_{1}-2, x_{2}\right)\right] \\
& +\frac{\lambda}{R} V_{n}\left(x_{1}, x_{2}\right)+\left(1-\frac{\mu_{1} m+\lambda}{R}\right) V_{n}\left(x_{1}-1, x_{2}\right) \\
& -\left\{\frac{\mu_{1} m}{R}\left[p V_{n}\left(x_{1}-1, x_{2}+k-1\right)+(1-p) V_{n}\left(x_{1}-1, x_{2}-1\right)\right]\right. \\
& \left.+\frac{\lambda}{R} V_{n}\left(x_{1}+1, x_{2}-1\right)+\left(1-\frac{\mu_{1} m+\lambda}{R}\right) V_{n}\left(x_{1}, x_{2}-1\right)\right\} \\
= & \frac{\mu_{1} m}{R}\left[p \mathcal{G}_{n}\left(x_{1}-1, x_{2}+k\right)+(1-p) \mathcal{G}_{n}\left(x_{1}-1, x_{2}\right)\right] \\
& +\frac{\lambda}{R} \mathcal{G}_{n}\left(x_{1}+1, x_{2}\right)+\left(1-\frac{\mu_{1} m+\lambda}{R}\right) \mathcal{G}_{n}\left(x_{1}, x_{2}\right) .
\end{aligned}
$$

The first and third equalities follow from Eq. (14). The second equality follows from Eqs. (44), (43), and (5).

Proof of Equation (23): If $x_{1} \leq m<x_{1}+x_{2}$,

$$
\begin{array}{ll}
g_{1}^{*}\left(x_{1}-1, x_{2}\right)=x_{1}-1, & g_{2}^{*}\left(x_{1}-1, x_{2}\right)=m-x_{1}+1, \\
g_{1}^{*}\left(x_{1}, x_{2}-1\right)=x_{1}, & g_{2}^{*}\left(x_{1}, x_{2}-1\right)=m-x_{1} .
\end{array}
$$


Then,

$$
\begin{aligned}
& \mathcal{G}_{n+1}\left(x_{1}, x_{2}\right)=V_{n+1}\left(x_{1}-1, x_{2}\right)-V_{n+1}\left(x_{1}, x_{2}-1\right) \\
& =\frac{\mu_{1}\left(x_{1}-1\right)}{R}\left[p V_{n}\left(x_{1}-2, x_{2}+k\right)+(1-p) V_{n}\left(x_{1}-2, x_{2}\right)\right] \\
& +\frac{\mu_{2}\left(m-x_{1}+1\right)}{R} V_{n}\left(x_{1}-1, x_{2}-1\right)+\frac{\lambda}{R} V_{n}\left(x_{1}, x_{2}\right) \\
& +\left(1-\frac{\mu_{1}\left(x_{1}-1\right)+\mu_{2}\left(m-x_{1}+1\right)+\lambda}{R}\right) V_{n}\left(x_{1}-1, x_{2}\right) \\
& -\left\{\frac{\mu_{1} x_{1}}{R}\left[p V_{n}\left(x_{1}-1, x_{2}+k-1\right)+(1-p) V_{n}\left(x_{1}-1, x_{2}-1\right)\right]\right. \\
& +\frac{\mu_{2}\left(m-x_{1}\right)}{R} V_{n}\left(x_{1}, x_{2}-2\right)+\frac{\lambda}{R} V_{n}\left(x_{1}, x_{2}\right) \\
& \left.+\left(1-\frac{\mu_{1} x_{1}+\mu_{2}\left(m-x_{1}\right)+\lambda}{R}\right) V_{n}\left(x_{1}, x_{2}-1\right)\right\} \\
& =\frac{\mu_{1}\left(x_{1}-1\right)}{R}\left[p \mathcal{G}_{n}\left(x_{1}-1, x_{2}+k\right)+(1-p) \mathcal{G}_{n}\left(x_{1}-1, x_{2}\right)\right] \\
& +\frac{\mu_{2}\left(m-x_{1}\right)}{R} V_{n}\left(x_{1}, x_{2}-1\right)+\frac{\lambda}{R} \mathcal{G}_{n}\left(x_{1}+1, x_{2}\right) \\
& +\left(1-\frac{\mu_{1}\left(x_{1}-1\right)+\mu_{2}\left(m-x_{1}\right)+\lambda}{R}\right) \mathcal{G}_{n}\left(x_{1}, x_{2}\right) \\
& -\frac{\mu_{1}}{R}\left[p V_{n}\left(x_{1}-1, x_{2}+k-1\right)+(1-p) V_{n}\left(x_{1}-1, x_{2}-1\right)-V_{n}\left(x_{1}, x_{2}-1\right)\right] \\
& +\frac{\mu_{2}}{R}\left[V_{n}\left(x_{1}-1, x_{2}-1\right)-V_{n}\left(x_{1}-1, x_{2}\right)\right] \\
& =\frac{\mu_{1} x_{1}-1}{R}\left[p \mathcal{G}_{n}\left(x_{1}-1, x_{2}+k\right)-(1-p) \mathcal{G}_{n}\left(x_{1}-1, x_{2}\right)\right] \\
& +\frac{\mu_{2}\left(m-x_{1}\right)}{R} V_{n}\left(x_{1}, x_{2}-1\right)+\frac{\lambda}{R} \mathcal{G}_{n}\left(x_{1}+1, x_{2}\right) \\
& +\left(1-\frac{\mu_{1}\left(x_{1}-1\right)+\mu_{2}\left(m-x_{1}\right)+\lambda}{R}\right) \mathcal{G}_{n}\left(x_{1}, x_{2}\right) \\
& -\frac{1}{R} V_{n}^{1}\left(x_{1}, x_{2}-1\right)+\frac{1}{R} V_{n}^{2}\left(x_{1}-1, x_{2}\right) \\
& \leq \frac{\mu_{1}\left(x_{1}-1\right)}{R}\left[p \mathcal{G}_{n}\left(x_{1}-1, x_{2}+k\right)+(1-p) \mathcal{G}_{n}\left(x_{1}-1, x_{2}\right)\right] \\
& +\frac{\mu_{2}\left(m-x_{1}\right)}{R} V_{n}\left(x_{1}, x_{2}-1\right)+\frac{\lambda}{R} \mathcal{G}_{n}\left(x_{1}+1, x_{2}\right) \\
& +\left(1-\frac{\mu_{1}\left(x_{1}-1\right)+\mu_{2}\left(m-x_{1}\right)+\lambda}{R}\right) \mathcal{G}_{n}\left(x_{1}, x_{2}\right)-\frac{\mu_{1}}{R} \mathcal{G}_{n}\left(x_{1}, x_{2}\right) \\
& =\frac{\mu_{1}\left(x_{1}-1\right)}{R}\left[p \mathcal{G}_{n}\left(x_{1}-1, x_{2}+k\right)+(1-p) \mathcal{G}_{n}\left(x_{1}-1, x_{2}\right)\right] \\
& +\frac{\mu_{2}\left(m-x_{1}\right)}{R} V_{n}\left(x_{1}, x_{2}-1\right)+\frac{\lambda}{R} \mathcal{G}_{n}\left(x_{1}+1, x_{2}\right) \\
& +\left(1-\frac{\mu_{1} x_{1}+\mu_{2}\left(m-x_{1}\right)+\lambda}{R}\right) \mathcal{G}_{n}\left(x_{1}, x_{2}\right) \text {. }
\end{aligned}
$$


The first and third equalities follow from the definition of $\mathcal{G}_{n+1}\left(x_{1}, x_{2}\right)$ and $\mathcal{G}_{n}\left(x_{1}, x_{2}\right)$. The second equality is a result of Eqs. (45), (43), and (5). The fourth equality follows from definitions (10) and (11). The inequality holds because of Fact 3 [Eq. (19)].

Proof of Equation (24): If $x_{1}+x_{2} \leq m$,

$$
\begin{array}{ll}
g_{1}^{*}\left(x_{1}-1, x_{2}\right)=x_{1}-1, & g_{2}^{*}\left(x_{1}-1, x_{2}\right)=x_{2}, \\
g_{1}^{*}\left(x_{1}, x_{2}-1\right)=x_{1}, & g_{2}^{*}\left(x_{1}, x_{2}-1\right)=x_{2}-1 .
\end{array}
$$

Then,

$$
\begin{aligned}
& \mathcal{G}_{n+1}\left(x_{1}, x_{2}\right)=V_{n+1}\left(x_{1}-1, x_{2}\right)-V_{n+1}\left(x_{1}, x_{2}-1\right) \\
& =\frac{\mu_{1}\left(x_{1}-1\right)}{R}\left[p V_{n}\left(x_{1}-2, x_{2}+k\right)+(1-p) V_{n}\left(x_{1}-2, x_{2}\right)\right] \\
& +\frac{\mu_{2} x_{2}}{R} V_{n}\left(x_{1}-1, x_{2}-1\right)+\frac{\lambda}{R} V_{n}\left(x_{1}, x_{2}\right) \\
& +\left(1-\frac{\mu_{1}\left(x_{1}-1\right)+\mu_{2} x_{2}+\lambda}{R}\right) V_{n}\left(x_{1}-1, x_{2}\right) \\
& -\left\{\frac{\mu_{1} x_{1}}{R}\left[p V_{n}\left(x_{1}-1, x_{2}+k-1\right)+(1-p) V_{n}\left(x_{1}-1, x_{2}-1\right)\right]\right. \\
& +\frac{\mu_{2}\left(x_{2}-1\right)}{R} V_{n}\left(x_{1}, x_{2}-2\right)+\frac{\lambda}{R} V_{n}\left(x_{1}, x_{2}\right) \\
& \left.+\left(1-\frac{\mu_{1} x_{1}+\mu_{2}\left(x_{2}-1\right)+\lambda}{R}\right) V_{n}\left(x_{1}, x_{2}-1\right)\right\} \\
& =\frac{\mu_{1}\left(x_{1}-1\right)}{R}\left[p \mathcal{G}_{n}\left(x_{1}-1, x_{2}+k\right)+(1-p) \mathcal{G}_{n}\left(x_{1}-1, x_{2}\right)\right] \\
& +\frac{\mu_{2}\left(x_{2}-1\right)}{R} \mathcal{G}_{n}\left(x_{1}, x_{2}-1\right)+\frac{\lambda}{R} \mathcal{G}_{n}\left(x_{1}+1, x_{2}\right) \\
& +\left(1-\frac{\mu_{1}\left(x_{1}-1\right)+\mu_{2}\left(x_{2}-1\right)+\lambda}{R}\right) \mathcal{G}_{n}\left(x_{1}, x_{2}\right) \\
& -\frac{\mu_{1}}{R}\left[p V_{n}\left(x_{1}-1, x_{2}+k-1\right)+(1-p) V_{n}\left(x_{1}-1, x_{2}-1\right)-V_{n}\left(x_{1}, x_{2}-1\right)\right] \\
& +\frac{\mu_{2}}{R}\left[V_{n}\left(x_{1}-1, x_{2}-1\right)-V_{n}\left(x_{1}-1, x_{2}\right)\right] \\
& =\frac{\mu_{1}\left(x_{1}-1\right)}{R}\left[p \mathcal{G}_{n}\left(x_{1}-1, x_{2}+k\right)+(1-p) \mathcal{G}_{n}\left(x_{1}-1, x_{2}\right)\right] \\
& +\frac{\mu_{2}\left(x_{2}-1\right)}{R} \mathcal{G}_{n}\left(x_{1}, x_{2}-1\right)+\frac{\lambda}{R} \mathcal{G}_{n}\left(x_{1}+1, x_{2}\right) \\
& +\left(1-\frac{\mu_{1}\left(x_{1}-1\right)+\mu_{2}\left(x_{2}-1\right)+\lambda}{R}\right) \mathcal{G}_{n}\left(x_{1}, x_{2}\right) \\
& -\frac{1}{R} V_{n}^{1}\left(x_{1}, x_{2}-1\right)+\frac{1}{R} V_{n}^{2}\left(x_{1}-1, x_{2}\right)
\end{aligned}
$$




$$
\begin{aligned}
\leq & \frac{\mu_{1}\left(x_{1}-1\right)}{R}\left[p \mathcal{G}_{n}\left(x_{1}-1, x_{2}+k\right)+(1-p) \mathcal{G}_{n}\left(x_{1}-1, x_{2}\right)\right] \\
& +\frac{\mu_{2}\left(x_{2}-1\right)}{R} \mathcal{G}_{n}\left(x_{1}, x_{2}-1\right)+\frac{\lambda}{R} \mathcal{G}_{n}\left(x_{1}+1, x_{2}\right) \\
& +\left(1-\frac{\mu_{1}\left(x_{1}-1\right)+\mu_{2}\left(x_{2}-1\right)+\lambda}{R}\right) \mathcal{G}_{n}\left(x_{1}, x_{2}\right)-\frac{\mu_{1}}{R} \mathcal{G}_{n}\left(x_{1}, x_{2}\right) \\
= & \frac{\mu_{1}\left(x_{1}-1\right)}{R}\left[p \mathcal{G}_{n}\left(x_{1}-1, x_{2}+k\right)+(1-p) \mathcal{G}_{n}\left(x_{1}-1, x_{2}\right)\right] \\
& +\frac{\mu_{2}\left(x_{2}-1\right)}{R} \mathcal{G}_{n}\left(x_{1}, x_{2}-1\right)+\frac{\lambda}{R} \mathcal{G}_{n}\left(x_{1}+1, x_{2}\right) \\
& +\left(1-\frac{\mu_{1} x_{1}+\mu_{2}\left(x_{2}-1\right)+\lambda}{R}\right) \mathcal{G}_{n}\left(x_{1}, x_{2}\right) .
\end{aligned}
$$

Again, the first and third equalities follow from the definitions of $\mathcal{G}_{n+1}\left(x_{1}, x_{2}\right)$ and $\mathcal{G}_{n}\left(x_{1}, x_{2}\right)$. The second equality is a result of Eqs. (46), (43), and (5). The fourth equality follows from definitions (10) and (11). The inequality holds because of Fact 3 [Eq. (19)].

Proof of Equation (25): If $x_{1}>m$,

$$
\begin{array}{ll}
g_{1}^{*}\left(x_{1}-1, x_{2}\right)=m, & g_{2}^{*}\left(x_{1}-1, x_{2}\right)=0, \\
g_{1}^{*}\left(x_{1}-1, x_{2}+k-1\right)=m, & g_{2}^{*}\left(x_{1}-1, x_{2}+k-1\right)=0, \\
g_{1}^{*}\left(x_{1}, x_{2}-1\right)=m, & g_{2}^{*}\left(x_{1}, x_{2}-1\right)=0 .
\end{array}
$$

Using Eqs. (43), (47), and (13) and the induction hypothesis on $\mathcal{F}_{n}\left(x_{1}, x_{2}\right)$, we obtain

$$
\begin{aligned}
\mathcal{F}_{n+1}\left(x_{1}, x_{2}\right)= & p V_{n+1}\left(x_{1}-1, x_{2}+k-1\right)+(1-p) V_{n+1}\left(x_{1}-1, x_{2}\right)-V_{n+1}\left(x_{1}, x_{2}-1\right) \\
=p & \left\{\frac{\mu_{1} m}{R}\left[p V_{n}\left(x_{1}-2, x_{2}+2 k-1\right)+(1-p) V_{n}\left(x_{1}-2, x_{2}+k-1\right)\right]\right. \\
& \left.+\frac{\lambda}{R} V_{n}\left(x_{1}, x_{2}+k-1\right)+\left(1-\frac{\mu_{1} m+\lambda}{R}\right) V_{n}\left(x_{1}-1, x_{2}+k-1\right)\right\} \\
& +(1-p)\left\{\frac{\mu_{1} m}{R}\left[p V_{n}\left(x_{1}-2, x_{2}+k\right)+(1-p) V_{n}\left(x_{1}-2, x_{2}\right)\right]\right. \\
& \left.\quad+\frac{\lambda}{R} V_{n}\left(x_{1}, x_{2}\right)+\left(1-\frac{\mu_{1} m+\lambda}{R}\right) V_{n}\left(x_{1}-1, x_{2}\right)\right\} \\
& -\left\{\frac{\mu_{1} m}{R}\left[p V_{n}\left(x_{1}-1, x_{2}+k-1\right)+(1-p) V_{n}\left(x_{1}-1, x_{2}-1\right)\right]\right. \\
& \left.\quad+\frac{\lambda}{R} V_{n}\left(x_{1}+1, x_{2}-1\right)+\left(1-\frac{\mu_{1} m+\lambda}{R}\right) V_{n}\left(x_{1}, x_{2}-1\right)\right\} \\
= & \frac{\mu_{1} m}{R}\left[p \mathcal{F}_{n}\left(x_{1}-1, x_{2}+k\right)+(1-p) \mathcal{F}_{n}\left(x_{1}-1, x_{2}\right)\right] \\
& +\frac{\lambda}{R} \mathcal{F}_{n}\left(x_{1}+1, x_{2}\right)+\left(1-\frac{\mu_{1} m+\lambda}{R}\right) \mathcal{F}_{n}\left(x_{1}, x_{2}\right) .
\end{aligned}
$$


The first and third equalities follow from Eq. (13). The second equality follows from Eqs. (47), (43), and (5).

Proof of Equation (26): If $x_{1} \leq m<x_{1}+x_{2}$,

$$
\begin{array}{ll}
g_{1}^{*}\left(x_{1}-1, x_{2}\right)=x_{1}-1 & g_{2}^{*}\left(x_{1}-1, x_{2}\right)=m-x_{1}+1 \\
g_{1}^{*}\left(x_{1}-1, x_{2}+k-1\right)=x_{1}-1 & g_{2}^{*}\left(x_{1}-1, x_{2}+k-1\right)=m-x_{1}+1 \\
g_{1}^{*}\left(x_{1}, x_{2}-1\right)=x_{1} & g_{2}^{*}\left(x_{1}, x_{2}-1\right)=m-x_{1} .
\end{array}
$$

Then

$$
\begin{aligned}
& \mathcal{F}_{n+1}\left(x_{1}, x_{2}\right)=p V_{n+1}\left(x_{1}-1, x_{2}+k-1\right)+(1-p) V_{n+1}\left(x_{1}-1, x_{2}\right)-V_{n+1}\left(x_{1}, x_{2}-1\right) \\
& =p\left\{\frac{\mu_{1}\left(x_{1}-1\right)}{R}\left[p V_{n}\left(x_{1}-2, x_{2}+k\right)+(1-p) V_{n}\left(x_{1}-2, x_{2}\right)\right]\right. \\
& +\frac{\mu_{2}\left(m-x_{1}+1\right)}{R} V_{n}\left(x_{1}-1, x_{2}-1\right)+\frac{\lambda}{R} V_{n}\left(x_{1}, x_{2}\right) \\
& \left.+\left(1-\frac{\mu_{1}\left(x_{1}-1\right)+\mu_{2}\left(m-x_{1}+1\right)+\lambda}{R}\right) V_{n}\left(x_{1}-1, x_{2}\right)\right\} \\
& +(1-p)\left\{\frac{\mu_{1}\left(x_{1}-1\right)}{R}\left[p V_{n}\left(x_{1}-2, x_{2}+k\right)+(1-p) V_{n}\left(x_{1}-2, x_{2}\right)\right]\right. \\
& +\frac{\mu_{2}\left(m-x_{1}+1\right)}{R} V_{n}\left(x_{1}-1, x_{2}-1\right)+\frac{\lambda}{R} V_{n}\left(x_{1}, x_{2}\right) \\
& \left.+\left(1-\frac{\mu_{1}\left(x_{1}-1\right)+\mu_{2}\left(m-x_{1}+1\right)+\lambda}{R}\right) V_{n}\left(x_{1}-1, x_{2}\right)\right\} \\
& -\left\{\frac{\mu_{1} x_{1}}{R}\left[p V_{n}\left(x_{1}-1, x_{2}+k-1\right)+(1-p) V_{n}\left(x_{1}-1, x_{2}-1\right)\right]\right. \\
& +\frac{\mu_{2}\left(m-x_{1}\right)}{R} V_{n}\left(x_{1}, x_{2}-2\right)+\frac{\lambda}{R} V_{n}\left(x_{1}, x_{2}\right) \\
& \left.+\left(1-\frac{\mu_{1} x_{1}+\mu_{2}\left(m-x_{1}\right)+\lambda}{R}\right) V_{n}\left(x_{1}, x_{2}-1\right)\right\} \\
& =\frac{\mu_{1}\left(x_{1}-1\right)}{R}\left[p \mathcal{F}_{n}\left(x_{1}-1, x_{2}+k\right)+(1-p) \mathcal{F}_{n}\left(x_{1}-1, x_{2}\right)\right] \\
& +\frac{\mu_{2}\left(m-x_{1}\right)}{R} \mathcal{F}_{n}\left(x_{1}, x_{2}-1\right)+\frac{\lambda}{R} \mathcal{F}_{n}\left(x_{1}+1, x_{2}\right) \\
& +\left(1-\frac{\mu_{1}\left(x_{1}-1\right)+\mu_{2}\left(m-x_{1}\right)+\lambda}{R}\right) \mathcal{F}_{n}\left(x_{1}, x_{2}\right) \\
& -\frac{\mu_{1}}{R}\left[p \mathcal{F}_{n}\left(x_{1}-1, x_{2}+k-1\right)+(1-p) V_{n}\left(x_{1}-1, x_{2}-1\right)-V_{n}\left(x_{1}, x_{2}-1\right)\right] \\
& +\frac{\mu_{2}}{R} p\left[V_{n}\left(x_{1}-1, x_{2}+k-2\right)-V_{n}\left(x_{1}-1, x_{2}+k-1\right)\right] \\
& +\frac{\mu_{2}}{R}(1-p)\left[V_{n}\left(x_{1}-1, x_{2}-1\right)-V_{n}\left(x_{1}-1, x_{2}\right)\right]
\end{aligned}
$$




$$
\begin{aligned}
& =\frac{\mu_{1}\left(x_{1}-1\right)}{R}\left[p \mathcal{F}_{n}\left(x_{1}-1, x_{2}+k\right)+(1-p) \mathcal{F}_{n}\left(x_{1}-1, x_{2}\right)\right] \\
& +\frac{\mu_{2}\left(m-x_{1}\right)}{R} \mathcal{F}_{n}\left(x_{1}, x_{2}-1\right)+\frac{\lambda}{R} \mathcal{F}_{n}\left(x_{1}+1, x_{2}\right) \\
& +\left(1-\frac{\mu_{1}\left(x_{1}-1\right)+\mu_{2}\left(m-x_{1}\right)+\lambda}{R}\right) \mathcal{F}_{n}\left(x_{1}, x_{2}\right) \\
& -\frac{1}{R} V_{n}^{1}\left(x_{1}, x_{2}-1\right)+\frac{p}{R} V_{n}^{2}\left(x_{1}-1, x_{2}+k-1\right)+\frac{1-p}{R} V_{n}^{2}\left(x_{1}-1, x_{2}\right) \\
& \leq \frac{\mu_{1}\left(x_{1}-1\right)}{R}\left[p \mathcal{F}_{n}\left(x_{1}-1, x_{2}+k\right)+(1-p) \mathcal{F}_{n}\left(x_{1}-1, x_{2}\right)\right] \\
& +\frac{\mu_{2}\left(m-x_{1}\right)}{R} \mathcal{F}_{n}\left(x_{1}, x_{2}-1\right)+\frac{\lambda}{R} \mathcal{F}_{n}\left(x_{1}+1, x_{2}\right) \\
& +\left(1-\frac{\mu_{1}\left(x_{1}-1\right)+\mu_{2}\left(m-x_{2}\right)+\lambda}{R}\right) \mathcal{F}_{n}\left(x_{1}, x_{2}\right) \\
& +\frac{\mu_{1}}{R}\left\{(1-p) \mathcal{G}_{n}\left(x_{1}, x_{2}-1\right)+p \mathcal{G}_{n}\left(x_{1}, x_{2}\right)+(1-p) \mathcal{F}_{n}\left(x_{1}, x_{2}-1\right)\right. \\
& \left.-(1-p) \mathcal{F}_{n}\left(x_{1}, x_{2}\right)\right\} \\
& =\frac{\mu_{1}\left(x_{1}-1\right)}{R}\left[p \mathcal{F}_{n}\left(x_{1}-1, x_{2}+k\right)+(1-p) \mathcal{F}_{n}\left(x_{1}-1, x_{2}\right)\right] \\
& +\frac{\mu_{2}\left(m-x_{1}\right)}{R} \mathcal{F}_{n}\left(x_{1}, x_{2}-1\right)+\frac{\lambda}{R} \mathcal{F}_{n}\left(x_{1}+1, x_{2}\right) \\
& +\left(1-\frac{\mu_{1}\left(x_{1}-1\right)+\mu_{2}\left(m-x_{1}\right)+\lambda}{R}\right) \mathcal{F}_{n}\left(x_{1}, x_{2}\right)-(1-p) \frac{\mu_{1}}{R} \mathcal{F}_{n}\left(x_{1}, x_{2}\right) \\
& +\frac{\mu_{1}}{R}\left\{(1-p) \mathcal{G}_{n}\left(x_{1}, x_{2}-1\right)+p \mathcal{G}_{n}\left(x_{1}, x_{2}\right)+(1-p) \mathcal{F}_{n}\left(x_{1}, x_{2}-1\right)\right\} \\
& =\frac{\mu_{1}\left(x_{1}-1\right)}{R}\left[p \mathcal{F}_{n}\left(x_{1}-1, x_{2}+k\right)+(1-p) \mathcal{F}_{n}\left(x_{1}-1, x_{2}\right)\right] \\
& +\frac{\mu_{2}\left(m-x_{1}\right)}{R} \mathcal{F}_{n}\left(x_{1}, x_{2}-1\right)+\frac{\lambda}{R} \mathcal{F}_{n}\left(x_{1}+1, x_{2}\right) \\
& +\left(1-\frac{\mu_{1}\left(x_{1}-1\right)+\mu_{2}\left(m-x_{1}\right)+\lambda}{R}\right) \mathcal{F}_{n}\left(x_{1}, x_{2}\right) \\
& +\frac{\mu_{1}}{R}\left\{(1-p) \mathcal{G}_{n}\left(x_{1}, x_{2}-1\right)+p \mathcal{G}_{n}\left(x_{1}, x_{2}\right)+(1-p) \mathcal{F}_{n}\left(x_{1}, x_{2}-1\right)\right\} .
\end{aligned}
$$

The first and third equalities follow from Eq. (13). The second equality follows from Eqs. (48), (43), and (5). The fourth equality follows from definitions (10) and (11). The last two equalities are simply grouping the terms. The inequality in Eq. (49) is a subsequent of Eq. (20) in Fact 4.

Proof of Equation (27): If $x_{1}+x_{2} \leq m$,

$$
\begin{array}{ll}
g_{1}^{*}\left(x_{1}-1, x_{2}\right)=x_{1}-1, & g_{2}^{*}\left(x_{1}-1, x_{2}\right)=x_{2}, \\
g_{1}^{*}\left(x_{1}-1, x_{2}+k-1\right)=x_{1}-1, & g_{2}^{*}\left(x_{1}-1, x_{2}+k-1\right)=x_{2}+N, \\
g_{1}^{*}\left(x_{1}, x_{2}-1\right)=x_{1}, & g_{2}^{*}\left(x_{1}, x_{2}-1\right)=x_{2}-1,
\end{array}
$$


where

$$
N=\min \left(k-1, m-x_{1}-x_{2}+1\right) .
$$

Thus,

$$
\begin{aligned}
& \mathcal{F}_{n+1}\left(x_{1}, x_{2}\right)=p V_{n+1}\left(x_{1}-1, x_{2}+k-1\right)+(1-p) V_{n+1}\left(x_{1}-1, x_{2}\right)-V_{n+1}\left(x_{1}, x_{2}-1\right) \\
& =p\left\{\frac{\mu_{1}\left(x_{1}-1\right)}{R}\left[p V_{n}\left(x_{1}-2, x_{2}+k\right)+(1-p) V_{n}\left(x_{1}-2, x_{2}\right)\right]\right. \\
& +\frac{\mu_{2}\left(x_{2}+n\right)}{R} V_{n}\left(x_{1}-1, x_{2}-1\right)+\frac{\lambda}{R} V_{n}\left(x_{1}, x_{2}\right) \\
& \left.+\left(1-\frac{\mu_{1}\left(x_{1}-1\right)+\mu_{2}\left(x_{2}+n\right)+\lambda}{R}\right) V_{n}\left(x_{1}-1, x_{2}\right)\right\} \\
& +(1-p)\left\{\frac{\mu_{1}\left(x_{1}-1\right)}{R}\left[p V_{n}\left(x_{1}-2, x_{2}+k\right)+(1-p) V_{n}\left(x_{1}-2, x_{2}\right)\right]\right. \\
& +\frac{\mu_{2}\left(x_{2}\right)}{R} V_{n}\left(x_{1}-1, x_{2}-1\right)+\frac{\lambda}{R} V_{n}\left(x_{1}, x_{2}\right) \\
& \left.-\left(1-\frac{\mu_{1}\left(x_{1}-1\right)+\mu_{2} x_{2}+\lambda}{R}\right) V_{n}\left(x_{1}-1, x_{2}\right)\right\} \\
& -\left\{\frac{\mu_{1} x_{1}}{R}\left[p V_{n}\left(x_{1}-1, x_{2}+k-1\right)+(1-p) V_{n}\left(x_{1}-1, x_{2}-1\right)\right]\right. \\
& +\frac{\mu_{2}\left(x_{2}-1\right)}{R} V_{n}\left(x_{1}, x_{2}-2\right)+\frac{\lambda}{R} V_{n}\left(x_{1}, x_{2}\right) \\
& \left.+\left(1-\frac{\mu_{1} x_{1}+\mu_{2}\left(x_{2}-1\right)+\lambda}{R}\right) V_{n}\left(x_{1}, x_{2}-1\right)\right\} \\
& =\frac{\mu_{1}\left(x_{1}-1\right)}{R}\left[p \mathcal{F}_{n}\left(x_{1}-1, x_{2}+k\right)+(1-p) \mathcal{F}_{n}\left(x_{1}-1, x_{2}\right)\right] \\
& +\frac{\mu_{2}\left(x_{2}-1\right)}{R} \mathcal{F}_{n}\left(x_{1}, x_{2}-1\right)+\frac{\lambda}{R} \mathcal{F}_{n}\left(x_{1}+1, x_{2}\right) \\
& +\left(1-\frac{\mu_{1}\left(x_{1}-1\right)+\mu_{2}\left(x_{2}-1\right)+\lambda}{R}\right) \mathcal{F}_{n}\left(x_{1}, x_{2}\right) \\
& -\frac{\mu_{1}}{R}\left[p V_{n}\left(x_{1}-1, x_{2}+k-1\right)+(1-p) V_{n}\left(x_{1}-1, x_{2}-1\right)-V_{n}\left(x_{1}, x_{2}-1\right)\right] \\
& +\frac{\mu_{2}}{R} p\left[V_{n}\left(x_{1}-1, x_{2}+k-2\right)-V_{n}\left(x_{1}-1, x_{2}+k-1\right)\right] \\
& +\frac{\mu_{2}}{R}(1-p)\left[V_{n}\left(x_{1}-1, x_{2}-1\right)-V_{n}\left(x_{1}-1, x_{2}\right)\right] \\
& +N \frac{\mu_{2}}{R}\left[V_{n}\left(x_{1}-1, x_{2}+k-2\right)-V_{n}\left(x_{1}-1, x_{2}+k-1\right)\right]
\end{aligned}
$$




$$
\begin{aligned}
& =\frac{\mu_{1}\left(x_{1}-1\right)}{R}\left[p \mathcal{F}_{n}\left(x_{1}-1, x_{2}+k\right)+(1-p) \mathcal{F}_{n}\left(x_{1}-1, x_{2}\right)\right] \\
& +\frac{\mu_{2}\left(x_{2}-1\right)}{R} \mathcal{F}_{n}\left(x_{1}, x_{2}-1\right)+\frac{\lambda}{R} \mathcal{F}_{n}\left(x_{1}+1, x_{2}\right) \\
& +\left(1-\frac{\mu_{1}\left(x_{1}-1\right)+\mu_{2}\left(x_{2}-1\right)+\lambda}{R}\right) \mathcal{F}_{n}\left(x_{1}, x_{2}\right) \\
& -\frac{1}{R} V_{n}^{1}\left(x_{1}, x_{2}-1\right)+\frac{p}{R} V_{n}^{2}\left(x_{1}-1, x_{2}+k-1\right)+\frac{1-p}{R} V_{n}^{2}\left(x_{1}-1, x_{2}\right) \\
& +N \frac{\mu_{2}}{R} V_{n}^{2}\left(x_{1}-1, x_{2}+k-1\right) \\
& \leq \frac{\mu_{1}\left(x_{1}-1\right)}{R}\left[p \mathcal{F}_{n}\left(x_{1}-1, x_{2}+k\right)+(1-p) \mathcal{F}_{n}\left(x_{1}-1, x_{2}\right)\right] \\
& +\frac{\mu_{2}\left(x_{2}-1\right)}{R} \mathcal{F}_{n}\left(x_{1}, x_{2}-1\right)+\frac{\lambda}{R} \mathcal{F}_{n}\left(x_{1}+1, x_{2}\right) \\
& +\left(1-\frac{\mu_{1}\left(x_{1}-1\right)+\mu_{2}\left(x_{2}-1\right)+\lambda}{R}\right) \mathcal{F}_{n}\left(x_{1}, x_{2}\right) \\
& -\frac{1}{R} V_{n}^{1}\left(x_{1}, x_{2}-1\right)+\frac{p}{R} V_{n}^{2}\left(x_{1}-1, x_{2}+k-1\right)+\frac{1-p}{R} V_{n}^{2}\left(x_{1}-1, x_{2}\right) \\
& \leq \frac{\mu_{1}\left(x_{1}-1\right)}{R}\left[p \mathcal{F}_{n}\left(x_{1}-1, x_{2}+k\right)+(1-p) \mathcal{F}_{n}\left(x_{1}-1, x_{2}\right)\right] \\
& +\frac{\mu_{2}\left(x_{2}-1\right)}{R} \mathcal{F}_{n}\left(x_{1}, x_{2}-1\right)+\frac{\lambda}{R} \mathcal{F}_{n}\left(x_{1}+1, x_{2}\right) \\
& +\left(1-\frac{\mu_{1}\left(x_{1}-1\right)+\mu_{2}\left(x_{2}-1\right)+\lambda}{R}\right) \mathcal{F}_{n}\left(x_{1}, x_{2}\right) \\
& +\frac{\mu_{1}}{R}\left\{(1-p) \mathcal{G}_{n}\left(x_{1}, x_{2}-1\right)+p \mathcal{G}_{n}\left(x_{1}, x_{2}\right)+(1-p) \mathcal{F}_{n}\left(x_{1}, x_{2}-1\right)\right. \\
& \left.-(1-p) \mathcal{F}_{n}\left(x_{1}, x_{2}\right)\right\} \\
& =\frac{\mu_{1}\left(x_{1}-1\right)}{R}\left[p \mathcal{F}_{n}\left(x_{1}-1, x_{2}+k\right)+(1-p) \mathcal{F}_{n}\left(x_{1}-1, x_{2}\right)\right] \\
& +\frac{\mu_{2}\left(x_{2}-1\right)}{R} \mathcal{F}_{n}\left(x_{1}, x_{2}-1\right)+\frac{\lambda}{R} \mathcal{F}_{n}\left(x_{1}+1, x_{2}\right) \\
& +\left(1-\frac{\mu_{1}\left(x_{1}-1\right)+\mu_{2}\left(x_{2}-1\right)+\lambda}{R}\right) \mathcal{F}_{n}\left(x_{1}, x_{2}\right) \\
& +\frac{\mu_{1}}{R}\left\{(1-p) \mathcal{G}_{n}\left(x_{1}, x_{2}-1\right)+p \mathcal{G}_{n}\left(x_{1}, x_{2}\right)+(1-p) \mathcal{F}_{n}\left(x_{1}, x_{2}-1\right)\right\} .
\end{aligned}
$$

The first and third equalities follow from Eq. (13). The second equality follows from Eqs. (50), (43), and (5). The fourth equality follows from definitions (10) and (11). The first inequality in Eq. (52) holds because of $N \geq 0$ [N is given by Eq. (51)] and the second inequality is a subsequent of Eq. (20) in Fact 4. 
Proof of Equation (28): If $x_{1}>m$,

$$
\begin{array}{ll}
g_{1}^{*}\left(x_{1}-1, x_{2}\right)=m, & g_{2}^{*}\left(x_{1}-1, x_{2}\right)=0, \\
g_{1}^{*}\left(x_{1}-1, x_{2}+k\right)=m, & g_{2}^{*}\left(x_{1}-1, x_{2}+k\right)=0, \\
g_{1}^{*}\left(x_{1}, x_{2}-1\right)=m, & g_{2}^{*}\left(x_{1}, x_{2}-1\right)=0, \\
g_{1}^{*}\left(x_{1}, x_{2}\right)=m, & g_{2}^{*}\left(x_{1}, x_{2}\right)=0 .
\end{array}
$$

Then

$$
\begin{aligned}
V_{n+1}^{1}\left(x_{1}, x_{2}\right)=\mu_{1}\left(p V_{n+1}\left(x_{1}-1, x_{2}+k\right)+(1-p) V_{n+1}\left(x_{1}-1, x_{2}\right)-V_{n+1}\left(x_{1}, x_{2}\right)\right) \\
=\mu_{1}\left(p \left\{\frac{\mu_{1} m}{R}\left[p V_{n}\left(x_{1}-2, x_{2}+2 k\right)+(1-p) V_{n}\left(x_{1}-2, x_{2}+k\right)\right]\right.\right. \\
\left.\quad+\frac{\lambda}{R} V_{n}\left(x_{1}, x_{2}+k\right)+\left(1-\frac{\mu_{1} m+\lambda}{R}\right) V_{n}\left(x_{1}-1, x_{2}+k\right)\right\} \\
+(1-p)\left\{\frac{\mu_{1} m}{R}\left[p V_{n}\left(x_{1}-2, x_{2}+k\right)+(1-p) V_{n}\left(x_{1}-2, x_{2}\right)\right]\right. \\
\left.\quad+\frac{\lambda}{R} V_{n}\left(x_{1}, x_{2}\right)+\left(1-\frac{\mu_{1} m+\lambda}{R}\right) V_{n}\left(x_{1}-1, x_{2}\right)\right\} \\
\quad-\left\{\frac{\mu_{1} m}{R}\left[p V_{n}\left(x_{1}-1, x_{2}+k\right)+(1-p) V_{n}\left(x_{1}-1, x_{2}\right)\right]\right. \\
\left.\left.+\frac{\lambda}{R} V_{n}\left(x_{1}+1, x_{2}\right)+\left(1-\frac{\mu_{1} m+\lambda}{R}\right) V_{n}\left(x_{1}, x_{2}\right)\right\}\right) \\
+\frac{\mu_{1} m}{R}\left[p V_{n}^{1}\left(x_{1}-1, x_{2}+k\right)+(1-p) V_{n}^{1}\left(x_{1}-1, x_{2}\right)\right] \\
+\frac{\lambda}{R} V_{n}^{1}\left(x_{1}+1, x_{2}\right)+\left(1-\frac{\mu_{1} m+\lambda}{R}\right) V_{n}^{1}\left(x_{1}, x_{2}\right) .
\end{aligned}
$$

The first and third equalities follow from Eq. (10). The second equality follows from Eqs. (53), (43), and (5).

$$
\begin{aligned}
V_{n+1}^{2}\left(x_{1}, x_{2}\right)=\mu_{2}( & \left.V_{n+1}\left(x_{1}, x_{2}-1\right)-V_{n+1}\left(x_{1}, x_{2}\right)\right) \\
=\mu_{2}( & \frac{\mu_{1} m}{R}\left[p V_{n}\left(x_{1}-1, x_{2}+k-1\right)+(1-p) V_{n}\left(x_{1}-1, x_{2}-1\right)\right] \\
+ & \frac{\lambda}{R} V_{n}\left(x_{1}+1, x_{2}-1\right)+\left(1-\frac{\mu_{1} m+\lambda}{R}\right) V_{n}\left(x_{1}, x_{2}-1\right) \\
& -\left\{\frac{\mu_{1} m}{R}\left[p V_{n}\left(x_{1}-1, x_{2}+k\right)+(1-p) V_{n}\left(x_{1}-1, x_{2}\right)\right]\right. \\
& \left.\left.\quad+\frac{\lambda}{R} V_{n}\left(x_{1}+1, x_{2}\right)+\left(1-\frac{\mu_{1} m+\lambda}{R}\right) V_{n}\left(x_{1}, x_{2}\right)\right\}\right)
\end{aligned}
$$




$$
\begin{aligned}
= & \frac{\mu_{1} m}{R}\left[p V_{n}^{2}\left(x_{1}-1, x_{2}+k\right)+(1-p) V_{n}^{2}\left(x_{1}-1, x_{2}\right)\right] \\
& +\frac{\lambda}{R} V_{n}^{2}\left(x_{1}+1, x_{2}\right)+\left(1-\frac{\mu_{1} m+\lambda}{R}\right) V_{n}^{2}\left(x_{1}, x_{2}\right) .
\end{aligned}
$$

The first and third equalities follow from Eq. (11). The second equality follows from Eqs. (53), (43), and (5).

Because of Eqs. (54) and (55), we can write

$$
\begin{aligned}
\mathcal{D}_{n+1}\left(x_{1}, x_{2}\right)= & \frac{\mu_{1} m}{R}\left[p V_{n}^{1}\left(x_{1}-1, x_{2}+k\right)+(1-p) V_{n}^{1}\left(x_{1}-1, x_{2}\right)\right] \\
& +\frac{\lambda}{R} V_{n}^{1}\left(x_{1}+1, x_{2}\right)+\left(1-\frac{\mu_{1} m+\lambda}{R}\right) V_{n}^{1}\left(x_{1}, x_{2}\right) \\
& -\left\{\frac{\mu_{1} m}{R}\left[p V_{n}^{2}\left(x_{1}-1, x_{2}+k\right)+(1-p) V_{n}^{2}\left(x_{1}-1, x_{2}\right)\right]\right. \\
& \left.+\frac{\lambda}{R} V_{n}^{2}\left(x_{1}+1, x_{2}\right)+\left(1-\frac{\mu_{1} m+\lambda}{R}\right) V_{n}^{2}\left(x_{1}, x_{2}\right)\right\} \\
= & \frac{\mu_{1} m}{R}\left[p \mathcal{D}_{n}\left(x_{1}-1, x_{2}+k\right)+(1-p) \mathcal{D}_{n}\left(x_{1}-1, x_{2}\right)\right] \\
& +\frac{\lambda}{R} \mathcal{D}_{n}\left(x_{1}+1, x_{2}\right)+\left(1-\frac{\mu_{1} m+\lambda}{R}\right) \mathcal{D}_{n}\left(x_{1}, x_{2}\right) .
\end{aligned}
$$

The first and third inequalities hold because of Eq. (12). The second equality holds because of Eqs. (54) and (55).

Proof of Equation (29): If $x_{1} \leq m<x_{1}+x_{2}$,

$$
\begin{array}{ll}
g_{1}^{*}\left(x_{1}-1, x_{2}\right)=x_{1}-1, & g_{2}^{*}\left(x_{1}-1, x_{2}\right)=m-x_{1}+1, \\
g_{1}^{*}\left(x_{1}-1, x_{2}+k\right)=x_{1}-1, & g_{2}^{*}\left(x_{1}-1, x_{2}+k\right)=m-x_{1}+1, \\
g_{1}^{*}\left(x_{1}, x_{2}-1\right)=x_{1}, & g_{2}^{*}\left(x_{1}, x_{2}-1\right)=m-x_{1}, \\
g_{1}^{*}\left(x_{1}, x_{2}\right)=x_{1}, & g_{2}^{*}\left(x_{1}, x_{2}\right)=m-x_{1} .
\end{array}
$$

Then

$$
\begin{array}{r}
V_{n+1}^{1}\left(x_{1}, x_{2}\right)=\mu_{1}\left(p V_{n+1}\left(x_{1}-1, x_{2}+k\right)+(1-p) V_{n+1}\left(x_{1}-1, x_{2}\right)-V_{n+1}\left(x_{1}, x_{2}\right)\right) \\
=\mu_{1}\left(p \left\{\frac{\mu_{1}\left(x_{1}-1\right)}{R}\left[p V_{n}\left(x_{1}-2, x_{2}+2 k\right)+(1-p) V_{n}\left(x_{1}-2, x_{2}+k\right)\right]\right.\right. \\
\quad+\frac{\mu_{2}\left(m-x_{1}+1\right)}{R} V_{n}\left(x_{1}-1, x_{2}+k-1\right)+\frac{\lambda}{R} V_{n}\left(x_{1}, x_{2}+k\right) \\
\left.+\left(1-\frac{\mu_{1}\left(x_{1}-1\right)+\mu_{2}\left(m-x_{1}+1\right)+\lambda}{R}\right) V_{n}\left(x_{1}-1, x_{2}+k\right)\right\}
\end{array}
$$




$$
\begin{gathered}
+(1-p)\left\{\frac{\mu_{1}\left(x_{1}-1\right)}{R}\left[p V_{n}\left(x_{1}-2, x_{2}+k\right)+(1-p) V_{n}\left(x_{1}-2, x_{2}\right)\right]\right. \\
+\frac{\mu_{2}\left(m-x_{1}+1\right)}{R} V_{n}\left(x_{1}-1, x_{2}-1\right) \frac{\lambda}{R} V_{n}\left(x_{1}, x_{2}\right) \\
\left.+\left(1-\frac{\mu_{1}\left(x_{1}-1\right)+\mu_{2}\left(m-x_{1}+1\right)+\lambda}{R}\right) V_{n}\left(x_{1}-1, x_{2}\right)\right\} \\
-\left\{\frac{\mu_{1} x_{1}}{R}\left[p V_{n}\left(x_{1}-1, x_{2}+k\right)+(1-p) V_{n}\left(x_{1}-1, x_{2}\right)\right]\right. \\
+\frac{\mu_{2}\left(m-x_{1}\right)}{R} V_{n}\left(x_{1}, x_{2}-1\right)+\frac{\lambda}{R} V_{n}\left(x_{1}+1, x_{2}\right) \\
\left.\left.+\left(1-\frac{\mu_{1} x_{1}+\mu_{2}\left(m-x_{1}\right)+\lambda}{R}\right) V_{n}\left(x_{1}, x_{2}\right)\right\}\right) \\
=\frac{\mu_{1}\left(x_{1}-1\right)}{R}\left[p V_{n}^{1}\left(x_{1}-1, x_{2}+k\right)+(1-p) V_{n}^{1}\left(x_{1}-1, x_{2}\right)\right] \\
+\frac{\mu_{2}\left(m-x_{1}\right)}{R} V_{n}^{1}\left(x_{1}, x_{2}-1\right)+\frac{\lambda}{R} V_{n}^{1}\left(x_{1}+1, x_{2}\right) \\
+\left(1-\frac{\mu_{1}\left(x_{1}-1\right)+\mu_{2}\left(m-x_{1}\right)+\lambda}{R}\right) V_{n}^{1}\left(x_{1}, x_{2}\right)-\frac{\mu_{1}}{R} V_{n}^{1}\left(x_{1}, x_{2}\right) \\
+\frac{\mu_{1}}{R}\left[p V_{n}^{2}\left(x_{1}-1, x_{2}+k\right)+(1-p) V_{n}^{2}\left(x_{1}-1, x_{2}+k\right)\right] .
\end{gathered}
$$

The first and third equalities follow from Eq. (10). The second equality follows from Eqs. (56), (43), and (5). Similarly,

$$
\begin{aligned}
V_{n+1}^{2}\left(x_{1}, x_{2}\right)= & \mu_{2}\left(V_{n+1}\left(x_{1}, x_{2}-1\right)-V_{n+1}\left(x_{1}, x_{2}\right)\right) \\
= & \mu_{2}\left(\frac{\mu_{1}\left(x_{1}-1\right)}{R}\left[p V_{n}\left(x_{1}-1, x_{2}+k-1\right)+(1-p) V_{n}\left(x_{1}-1, x_{2}-1\right)\right]\right. \\
& +\frac{\mu_{2}\left(m-x_{1}\right)}{R} V_{n}\left(x_{1}, x_{2}-2\right)+\frac{\lambda}{R} V_{n}\left(x_{1}+1, x_{2}-1\right) \\
& +\left(1-\frac{\left.\mu_{1} x_{1}+\mu_{2}\left(m-x_{1}\right)+\lambda\right) V_{n}\left(x_{1}, x_{2}-1\right)}{R}\right. \\
& -\left\{\frac{\mu_{1} x_{1}}{R}\left[p V_{n}\left(x_{1}-1, x_{2}+k\right)+(1-p) V_{n}\left(x_{1}-1, x_{2}\right)\right]\right. \\
& +\frac{\mu_{2}\left(m-x_{1}\right)}{R} V_{n}\left(x_{1}, x_{2}-1\right) \frac{\lambda}{R} V_{n}\left(x_{1}+1, x_{2}\right) \\
& \left.\left.+\left(1-\frac{\mu_{1} x_{1}+\mu_{2}\left(m-x_{1}\right)+\lambda}{R}\right) V_{n}\left(x_{1}, x_{2}\right)\right\}\right) \\
+ & \frac{\mu_{1} x_{1}}{R}\left[p V_{n}^{2}\left(x_{1}-1, x_{2}+k\right)+(1-p) V_{n}^{2}\left(x_{1}-1, x_{2}\right)\right] \\
+ & \frac{\mu_{2}\left(m-x_{1}\right)}{R} V_{n}^{2}\left(x_{1}, x_{2}-1\right)+\frac{\lambda}{R} V_{n}^{2}\left(x_{1}+1, x_{2}\right) \\
+ & \left(1-\frac{\mu_{1} x_{1}+\mu_{2}\left(m-x_{1}\right)+\lambda}{R}\right) V_{n}^{2}\left(x_{1}, x_{2}\right) .
\end{aligned}
$$


The first and third equalities follow from Eq. (11). The second equality follows from Eqs. (56), (43), and (5).

From Eqs. (57), (58), and (12), we have

$$
\begin{aligned}
& \mathcal{D}_{n+1}\left(x_{1}, x_{2}\right)=V_{n+1}^{1}\left(x_{1}, x_{2}\right)-V_{n+1}^{2}\left(x_{1}, x_{2}\right) \\
& =\frac{\mu_{1}\left(x_{1}-1\right)}{R}\left[p V_{n}^{1}\left(x_{1}-1, x_{2}+k\right)+(1-p) V_{n}^{1}\left(x_{1}-1, x_{2}\right)\right] \\
& +\frac{\mu_{2}\left(m-x_{1}\right)}{R} V_{n}^{1}\left(x_{1}, x_{2}-1\right)+\frac{\lambda}{R} V_{n}^{1}\left(x_{1}+1, x_{2}\right) \\
& +\left(1-\frac{\mu_{1}\left(x_{1}-1\right)+\mu_{2}\left(m-x_{1}\right)+\lambda}{R}\right) V_{n}^{1}\left(x_{1}, x_{2}\right)-\frac{\mu_{1}}{R} V_{n}^{1}\left(x_{1}, x_{2}\right) \\
& +\frac{\mu_{2}}{R}\left[p V_{n}^{2}\left(x_{1}-1, x_{2}+k\right)+(1-p) V_{n}^{2}\left(x_{1}-1, x_{2}\right)\right] \\
& -\left\{\frac{\mu_{1} x_{1}}{R}\left[p V_{n}^{2}\left(x_{1}-1, x_{2}+k\right)+(1-p) V_{n}^{2}\left(x_{1}-1, x_{2}\right)\right]\right. \\
& +\frac{\mu_{2}\left(m-x_{1}\right)}{R} V_{n}^{2}\left(x_{1}, x_{2}-1\right)+\frac{\lambda}{R} V_{n}^{2}\left(x_{1}+1, x_{2}\right) \\
& \left.+\left(1-\frac{\mu_{1} x_{1}+\mu_{2}\left(m-x_{1}\right)+\lambda}{R}\right) V_{n}^{2}\left(x_{1}, x_{2}\right)\right\} \\
& =\frac{\mu_{1}\left(x_{1}-1\right)}{R}\left[p \mathcal{D}_{n}\left(x_{1}-1, x_{2}+k\right)+(1-p) \mathcal{D}_{n}\left(x_{1}-1, x_{2}\right)\right] \\
& +\frac{\mu_{2}\left(m-x_{1}\right)}{R} \mathcal{D}_{n}\left(x_{1}, x_{2}-1\right)+\frac{\lambda}{R} \mathcal{D}_{n}\left(x_{1}+1, x_{2}\right) \\
& +\left(1-\frac{\mu_{1}\left(x_{1}-1\right)+\mu_{2}\left(m-x_{1}\right)+\lambda}{R}\right) \mathcal{D}_{n}\left(x_{1}, x_{2}\right) \\
& -\frac{\mu_{1}}{R} V_{n}^{1}\left(x_{1}, x_{2}\right)+\frac{\mu_{1}}{R} V_{n}^{2}\left(x_{1}, x_{2}\right) \\
& =\frac{\mu_{1}\left(x_{1}-1\right)}{R}\left[p \mathcal{D}_{n}\left(x_{1}-1, x_{2}+k\right)+(1-p) \mathcal{D}_{n}\left(x_{1}-1, x_{2}\right)\right] \\
& +\frac{\mu_{2}\left(m-x_{1}\right)}{R} \mathcal{D}_{n}\left(x_{1}, x_{2}-1\right)+\frac{\lambda}{R} \mathcal{D}_{n}\left(x_{1}+1, x_{2}\right) \\
& +\left(1-\frac{\mu_{1} x_{1}+\mu_{2}\left(m-x_{1}\right)+\lambda}{R}\right) \mathcal{D}_{n}\left(x_{1}, x_{2}\right) .
\end{aligned}
$$

The first, third, and fourth equalities hold because of Eq. (12). The second equality holds because of Eqs. (57) and (58). 
Proof of Equation (30): If $x_{1}+x_{2} \leq m$,

$$
\begin{array}{ll}
g_{1}^{*}\left(x_{1}-1, x_{2}\right)=x_{1}-1, & g_{2}^{*}\left(x_{1}-1, x_{2}\right)=x_{2}, \\
g_{1}^{*}\left(x_{1}-1, x_{2}+k\right)=x_{1}-1, & g_{2}^{*}\left(x_{1}-1, x_{2}+k\right)=x_{2}+L, \\
g_{1}^{*}\left(x_{1}, x_{2}-1\right)=x_{1}, & g_{2}^{*}\left(x_{1}, x_{2}-1\right)=x_{2}-1, \\
g_{1}^{*}\left(x_{1}, x_{2}\right)=x_{1}, & g_{2}^{*}\left(x_{1}, x_{2}\right)=x_{2},
\end{array}
$$

where

$$
L=\min \left(k, m-x_{1}-x_{2}+1\right) .
$$

Then

$$
\begin{aligned}
& V_{n+1}^{1}\left(x_{1}, x_{2}\right)=\mu_{1}\left(p V_{n+1}\left(x_{1}-1, x_{2}+k\right)+(1-p) V_{n+1}\left(x_{1}-1, x_{2}\right)-V_{n+1}\left(x_{1}, x_{2}\right)\right) \\
& =\mu_{1}\left(p \left\{\frac{\mu_{1}\left(x_{1}-1\right)}{R}\left[p V_{n}\left(x_{1}-2, x_{2}+2 k\right)+(1-p) V_{n}\left(x_{1}-2, x_{2}+k\right)\right]\right.\right. \\
& +\frac{\mu_{2}(x+L)}{R} V_{n}\left(x_{1}-1, x_{2}+k-1\right)+\frac{\lambda}{R} V_{n}\left(x_{1}, x_{2}+k\right) \\
& \left.+\left(1-\frac{\mu_{1}\left(x_{1}-1\right)+\mu_{2}\left(x_{2}+L\right)+\lambda}{R}\right) V_{n}\left(x_{1}-1, x_{2}+k\right)\right\} \\
& +(1-p)\left\{\frac { \mu _ { 1 } ( x - 1 - 1 ) } { R } \left[p V_{n}\left(x_{1}-2, x_{2}+k\right)\right.\right. \\
& \left.+(1-p) V_{n}\left(x_{1}-2, x_{2}\right)\right] \\
& +\frac{\mu_{2} x_{2}}{R} V_{n}\left(x_{1}-1, x_{2}-1\right) \frac{\lambda}{R} V_{n}\left(x_{1}, x_{2}\right) \\
& \left.+\left(1-\frac{\mu_{1}\left(x_{1}-1\right)+\mu_{2} x_{2}+\lambda}{R}\right) V_{n}\left(x_{1}-1, x_{2}\right)\right\} \\
& -\left\{\frac{\mu_{1} x_{1}}{R}\left[p V_{n}\left(x_{1}-1, x_{2}+k\right)+(1-p) V_{n}\left(x_{1}-1, x_{2}\right)\right]\right. \\
& +\frac{\mu_{2} x_{2}}{R} V_{n}\left(x_{1}, x_{2}-1\right)+\frac{\lambda}{R} V_{n}\left(x_{1}+1, x_{2}\right) \\
& \left.\left.+\left(1-\frac{\mu_{1} x_{1}+\mu_{2} x_{2}+\lambda}{R}\right) V_{n}\left(x_{1}, x_{2}\right)\right\}\right) \\
& =\frac{\mu_{1}\left(x_{1}-1\right)}{R}\left[p V_{n}^{1}\left(x_{1}-1, x_{2}+k\right)+(1-p) V_{n}^{1}\left(x_{1}-1, x_{2}\right)\right] \\
& +\frac{\mu_{2} x_{2}}{R} V_{n}^{1}\left(x_{1}, x_{2}-1\right)+\frac{\lambda}{R} V_{n}^{1}\left(x_{1}+1, x_{2}\right) \\
& +\left(1-\frac{\mu_{1}\left(x_{1}-1\right)+\mu_{2}\left(m-x_{1}\right)+\lambda}{R}\right) V_{n}^{1}\left(x_{1}, x_{2}\right)-\frac{\mu_{1}}{R} V_{n}^{1}\left(x_{1}, x_{2}\right) \\
& +\frac{\mu_{1} L}{R} p V_{n}^{2}\left(x_{1}-1, x_{2}+k\right) \text {. }
\end{aligned}
$$


The first and third equalities follow from Eq. (10). The second equality follows from Eqs. (56), (43), and (5).

$$
\begin{aligned}
V_{n+1}^{2}\left(x_{1}, x_{2}\right)= & \mu_{2}\left(V_{n+1}\left(x_{1}, x_{2}-1\right)-V_{n+1}\left(x_{1}, x_{2}\right)\right) \\
= & \mu_{2}\left(\frac{\mu_{1} x_{1}}{R}\left[p V_{n}\left(x_{1}-1, x_{2}+k-1\right)+(1-p) V_{n}\left(x_{1}-1, x_{2}-1\right)\right]\right. \\
& +\frac{\mu_{2}\left(x_{2}-1\right)}{R} V_{n}\left(x_{1}, x_{2}-2\right)+\frac{\lambda}{R} V_{n}\left(x_{1}+1, x_{2}-1\right) \\
& +\left(1-\frac{\left.\mu_{1} x_{1}+\mu_{2}\left(x_{2}-1\right)+\lambda\right) V_{n}\left(x_{1}, x_{2}-1\right)}{R}\right. \\
& -\left\{\frac{\mu_{1} x_{1}}{R}\left[p V_{n}\left(x_{1}-1, x_{2}+k\right)+(1-p) V_{n}\left(x_{1}-1, x_{2}\right)\right]\right. \\
& +\frac{\mu_{2} x_{2}}{R} V_{n}\left(x_{1}, x_{2}-1\right)+\frac{\lambda}{R} V_{n}\left(x_{1}+1, x_{2}\right) \\
+ & \left.\left.+\left(1-\frac{\mu_{1} x_{1}+\mu_{2} x_{2}+\lambda}{R}\right) V_{n}\left(x_{1}, x_{2}\right)\right\}\right) \\
& +\frac{\mu_{1} x_{1}}{R}\left[p V_{n}^{2}\left(x_{1}-1, x_{2}+k\right)+(1-p) V_{n}^{2}\left(x_{1}-1, x_{2}\right)\right] \\
+ & \left(1-\frac{\left.\mu_{2}-1\right)}{R} V_{n}^{2}\left(x_{1}, x_{2}-1\right)+\frac{\lambda}{R} V_{n}^{2}\left(x_{1}+1, x_{2}\right)\right. \\
+ & \mu_{2}\left(x_{2}-1\right)+\lambda \\
R & V_{n}^{2}\left(x_{1}, x_{2}\right)-\frac{\mu_{2}}{R} V_{n}^{2}\left(x_{1}, x_{2}\right) .
\end{aligned}
$$

The first and third equalities follow from Eq. (11). The second equality follows from Eqs. (59), (43), and (5). Now we can write

$$
\begin{aligned}
\mathcal{D}_{n+1}\left(x_{1}, x_{2}\right)= & V_{n+1}^{1}\left(x_{1}, x_{2}\right)-V_{n+1}^{2}\left(x_{1}, x_{2}\right) \\
= & \frac{\mu_{1}\left(x_{1}-1\right)}{R}\left[p V_{n}^{1}\left(x_{1}-1, x_{2}+k\right)+(1-p) V_{n}^{1}\left(x_{1}-1, x_{2}\right)\right] \\
& +\frac{\mu_{2} x_{2}}{R} V_{n}^{1}\left(x_{1}, x_{2}-1\right)+\frac{\lambda}{R} V_{n}^{1}\left(x_{1}+1, x_{2}\right) \\
& +\left(1-\frac{\mu_{1}\left(x_{1}-1\right)+\mu_{2}\left(m-x_{1}\right)+\lambda}{R}\right) V_{n}^{1}\left(x_{1}, x_{2}\right)-\frac{\mu_{1}}{R} V_{n}^{1}\left(x_{1}, x_{2}\right) \\
& +\frac{\mu_{1} L}{R} p V_{n}^{2}\left(x_{1}-1, x_{2}+k\right) \\
& -\left\{\frac{\mu_{1} x_{1}}{R}\left[p V_{n}^{2}\left(x_{1}-1, x_{2}+k\right)+(1-p) V_{n}^{2}\left(x_{1}-1, x_{2}\right)\right]\right. \\
& \quad+\frac{\mu_{2}\left(x_{2}-1\right)}{R} V_{n}^{2}\left(x_{1}, x_{2}-1\right)+\frac{\lambda}{R} V_{n}^{2}\left(x_{1}+1, x_{2}\right) \\
& \left.\quad+\left(1-\frac{\mu_{1} x_{1}+\mu_{2}\left(x_{2}-1\right)+\lambda}{R}\right) V_{n}^{2}\left(x_{1}, x_{2}\right)-\frac{\mu_{2}}{R} V_{n}^{2}\left(x_{1}, x_{2}\right)\right\}
\end{aligned}
$$




$$
\begin{aligned}
& =\frac{\mu_{1}\left(x_{1}-1\right)}{R}\left[p \mathcal{D}_{n}\left(x_{1}-1, x_{2}+k\right)+(1-p) \mathcal{D}_{n}\left(x_{1}-1, x_{2}\right)\right] \\
& +\frac{\mu_{2}\left(x_{2}-1\right)}{R} \mathcal{D}_{n}\left(x_{1}, x_{2}-1\right)+\frac{\lambda}{R} \mathcal{D}_{n}\left(x_{1}+1, x_{2}\right) \\
& +\left(1-\frac{\mu_{1}\left(x_{1}-1\right)+\mu_{2}\left(x_{2}-1\right)+\lambda}{R}\right) \mathcal{D}_{n}\left(x_{1}, x_{2}\right)-\frac{\mu_{1}}{R} V_{n}^{1}\left(x_{1}, x_{2}\right) \\
& +\frac{\mu_{2}}{R}\left[V_{n}^{1}\left(x_{1}, x_{2}-1\right)-V_{n}^{1}\left(x_{1}, x_{2}\right)\right]+\frac{\mu_{2}}{R} V_{n}^{2}\left(x_{1}, x_{2}\right) \\
& +p \frac{\mu_{1} L}{R} V_{n}^{2}\left(x_{1}-1, x_{2}+k\right)+\frac{\mu_{1}}{R} V_{n}^{2}\left(x_{1}, x_{2}\right) \\
& -\frac{\mu_{1}}{R}\left[p V_{n}^{2}\left(x_{1}-1, x_{2}+k\right)+(1-p) V_{n}^{2}\left(x_{1}-1, x_{2}\right)\right] \\
& =\frac{\mu_{1}\left(x_{1}-1\right)}{R}\left[p \mathcal{D}_{n}\left(x_{1}-1, x_{2}+k\right)+(1-p) \mathcal{D}_{n}\left(x_{1}-1, x_{2}\right)\right] \\
& +\frac{\mu_{2}\left(x_{2}-1\right)}{R} \mathcal{D}_{n}\left(x_{1}, x_{2}-1\right)+\frac{\lambda}{R} \mathcal{D}_{n}\left(x_{1}+1, x_{2}\right) \\
& +\left(1-\frac{\mu_{1} x_{1}+\mu_{2} x_{2}+\lambda}{R}\right) \mathcal{D}_{n}\left(x_{1}, x_{2}\right)+p \frac{\mu_{1}(L-1)}{R} V_{n}^{2}\left(x_{1}-1, x_{2}+k\right) \\
& +\frac{\mu_{2}}{R} V_{n}^{1}\left(x_{1}, x_{2}-1\right)-\frac{\mu_{1}}{R} V_{n}^{2}\left(x_{1}-1, x_{2}\right) \\
& =\frac{\mu_{1}\left(x_{1}-1\right)}{R}\left[p \mathcal{D}_{n}\left(x_{1}-1, x_{2}+k\right)+(1-p) \mathcal{D}_{n}\left(x_{1}-1, x_{2}\right)\right] \\
& +\frac{\mu_{2}\left(x_{2}-1\right)}{R} \mathcal{D}_{n}\left(x_{1}, x_{2}-1\right)+\frac{\lambda}{R} \mathcal{D}_{n}\left(x_{1}+1, x_{2}\right) \\
& +p \frac{\mu_{1}(L-1)}{R} V_{n}^{2}\left(x_{1}-1, x_{2}+k\right) \\
& +\left(1-\frac{\mu_{1} x_{1}+\mu_{2} x_{2}+\lambda}{R}\right) \mathcal{D}_{n}\left(x_{1}, x_{2}\right)+\frac{\mu_{1} \mu_{2}}{R} \mathcal{F}_{n}\left(x_{1}, x_{2}\right) \\
& \leq \frac{\mu_{1}\left(x_{1}-1\right)}{R}\left[p \mathcal{D}_{n}\left(x_{1}-1, x_{2}+k\right)+(1-p) \mathcal{D}_{n}\left(x_{1}-1, x_{2}\right)\right] \\
& +\frac{\mu_{2}\left(x_{2}-1\right)}{R} \mathcal{D}_{n}\left(x_{1}, x_{2}-1\right)+\frac{\lambda}{R} \mathcal{D}_{n}\left(x_{1}+1, x_{2}\right) \\
& +\left(1-\frac{\mu_{1} x_{1}+\mu_{2} x_{2}+\lambda}{R}\right) \mathcal{D}_{n}\left(x_{1}, x_{2}\right)+\frac{\mu_{1} \mu_{2}}{R} \mathcal{F}_{n}\left(x_{1}, x_{2}\right) .
\end{aligned}
$$

The first and third equalities hold because of Eq. (12). The second equality holds because of Eqs. (61) and (62). The fifth equality follows from Eq. (13). The inequality holds because of Eq. (18) and $L \geq 1$. 


\section{APPENDIX B \\ Outline of the Proof of Theorem 3}

In this appendix, we provide the reader with the essence of the proof of Theorem 3. First define

Condition 3:

$$
p \leq \frac{\mu_{1}-\mu_{2}}{\mu_{1}}
$$

as the complement of Condition 2. Theorem 3 can be proved by the same argument as in Section 2.2; that is, Problem (P) can be reduced to proving the optimality of $\tilde{g}$ under Condition 3 for the $n$-stage problem described by Eqs. (7)-(9). To proceed with the proof, we establish an induction similar to that of Section 2.3.1. Note that since $k=1, \mathcal{G}_{n}\left(x_{1}, x_{2}\right)=$ $\mathcal{F}_{n}\left(x_{1}, x_{2}\right)$ and the induction hypotheses for stage $n$ will be

$\left(\mathcal{H}^{\prime} 0\right)_{n}$ : policy $\tilde{g}$, which gives priority to $Q_{2}$, is optimal at stage $n$.

$\left(\mathcal{H}^{\prime} 1\right)_{n}: \mathcal{D}_{n}\left(x_{1}, x_{2}\right) \geq 0$ for every $x_{1} \geq 0, x_{2} \geq 0$.

$\left(\mathcal{H}^{\prime} 2\right)_{n}: \mathcal{G}_{n}\left(x_{1}, x_{2}\right) \geq 0$ for every $x_{1} \geq 0, x_{2} \geq 0$.

The basis of induction is verified through the same argument as in Section 2.3.2. Assuming that the induction hypotheses are valid at stage $n$, we use a number of facts and lemmas to prove the validity of $\left(\mathcal{H}^{\prime} 0\right)_{n+1}-\left(\mathcal{H}^{\prime} 2\right)_{n+1}$. These facts and lemmas, which are stated below, and their proofs are very similar to those of Section 2.3.2.

Fact 5: For every $n \geq 1, x_{1} \geq 0$, and $x_{2} \geq 0$, the following relations hold:

$$
\begin{aligned}
& V_{n}\left(x_{1}+1, x_{2}\right) \geq V_{n}\left(x_{1}, x_{2}\right), \\
& V_{n}\left(x_{1}, x_{2}+1\right) \geq V_{n}\left(x_{1}, x_{2}\right) .
\end{aligned}
$$

Fact 6: For every $n \geq 1, x_{1} \geq 0$, and $x_{2} \geq 0$, the following relations hold:

$$
\begin{aligned}
& V_{n}^{1}\left(x_{1}, x_{2}\right) \leq 0, \\
& V_{n}^{2}\left(x_{1}, x_{2}\right) \leq 0 .
\end{aligned}
$$

Fact 7: Under Condition 3 [i.e., $p \leq\left(\mu_{1}-\mu_{2}\right) / \mu_{1}$ ],

$$
V_{n}^{1}\left(x_{1}, x_{2}-1\right)-V_{n}^{2}\left(x_{1}-1, x_{2}\right) \leq \mu_{1} \mathcal{G}_{n}\left(x_{1}, x_{2}\right) .
$$

Lemma 6: Assume Conditions 1 and 3 hold. If $\mathcal{D}_{n}\left(x_{1}, x_{2}\right) \geq 0$ for all $x_{1} \geq 0, x_{2} \geq 0$, then $\tilde{g}$ is optimal at stage $n+1$.

Lemma 7: Assume that Conditions 1 and 3 hold. If $\tilde{g}$ is optimal at stage $n+1$ and $\mathcal{G}_{n}\left(x_{1}, x_{2}\right) \geq$ 0 for all $x_{1}, x_{2}$, then $\mathcal{G}_{n+1}\left(x_{1}, x_{2}\right) \geq 0$.

Lemma 8: Assume that Conditions 1 and 3 hold. If $\tilde{g}$ is optimal at stage $n+1, \mathcal{G}_{n}\left(x_{1}, x_{2}\right) \geq$ 0 , and $\mathcal{D}_{n}\left(x_{1}, x_{2}\right) \geq 0$ for all $x_{1}, x_{2}$, then $\mathcal{D}_{n+1}\left(x_{1}, x_{2}\right) \geq 0$. 